LA-14452

Approved for public release;

distribution is unlimited.

\title{
Analytic Solutions of an Unclassified Artifact
}

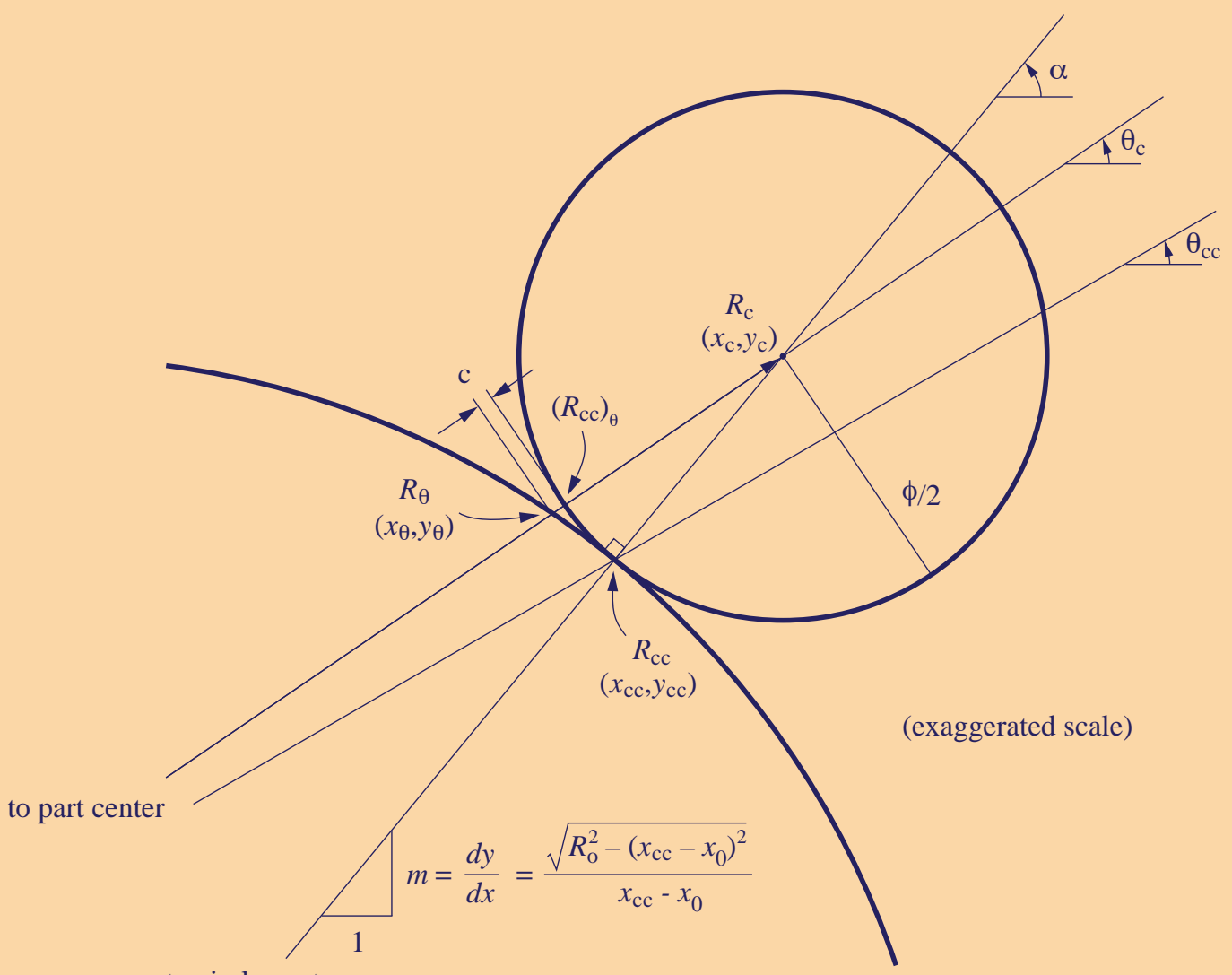

to circle center

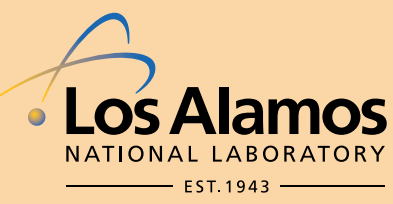


Funding for this report was provided by the U.S. Department of Energy.

Edited by Craig Carmer of Group IRM-CAS.

Los Alamos National Laboratory, an Affirmative Action/ Equal Opportunity Employer, is operated by Los Alamos National Security, LLC, for the National Nuclear Security Administration of the U.S. Department of Energy under contract DE-AC52-06NA25396.

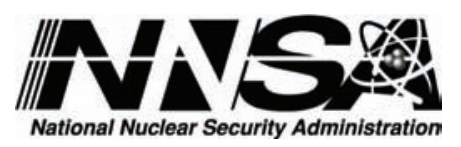

This report was prepared as an account of work sponsored by an agency of the U.S. Government. Neither Los Alamos National Security, LLC, the U.S. Government nor any agency thereof, nor any of their employees make any warranty, express or implied, or assume any legal liability or responsibility for the accuracy, completeness, or usefulness of any information, apparatus, product, or process disclosed, or represent that its use would not infringe privately owned rights. Reference herein to any specific commercial product, process, or service by trade name, trademark, manufacturer, or otherwise does not necessarily constitute or imply its endorsement, recommendation, or favoring by Los Alamos National Security, LLC, the U.S. Government, or any agency thereof. The views and opinions of authors expressed herein do not necessarily state or reflect those of Los Alamos National Security, LLC, the U.S. Government, or any agency thereof. Los Alamos National Laboratory strongly supports academic freedom and a researcher's right to publish; as an institution, however, the Laboratory does not endorse the viewpoint of a publication or guarantee its technical correctness. 
LA-14452

Issued: March 2012

\section{Analytic Solutions of an Unclassified Artifact}

Bruce C. Trent, W-2 
Analytic Solutions of an Unclassified Artifact 


\section{Contents}

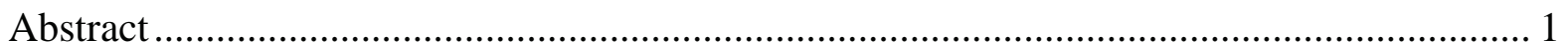

Chapter 1. A nalytic part definition ............................................................................. 3

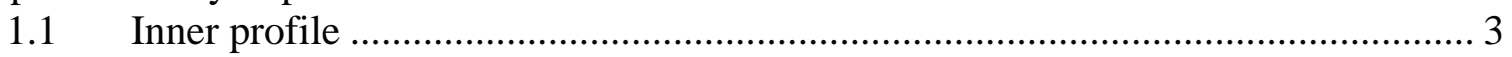

1.2 Outer profile

Chapter 2. Definition of the inspection sphere ……......................................................... 11

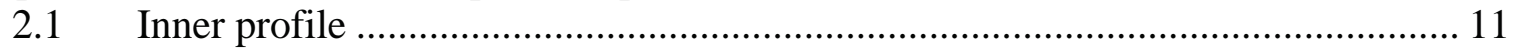

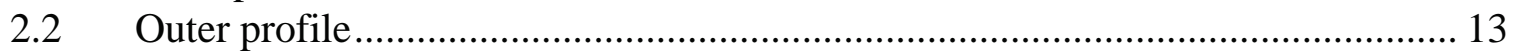

Chapter 3. Simultaneous equations for the contact point on the circular sections ................ 15

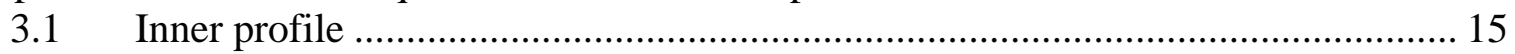

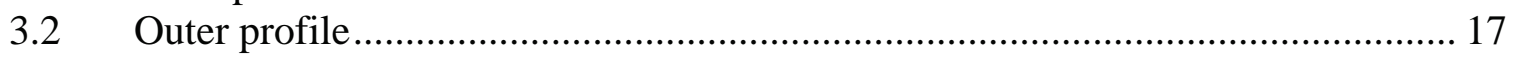

Chapter 4. Newton-Raphson solution for the circular sections ........................................... 21

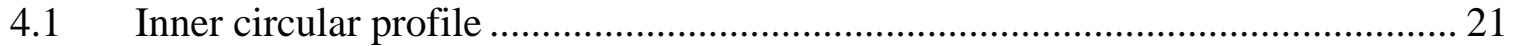

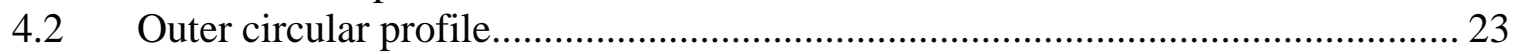

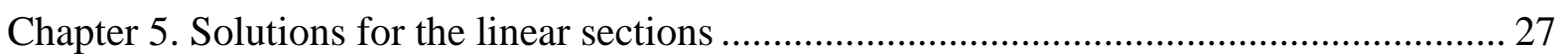

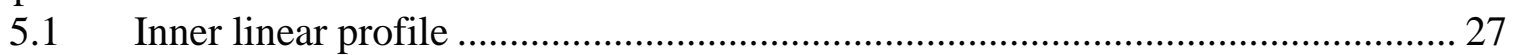

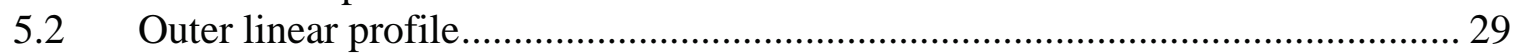

5.3 Solution for minimum component values................................................... 33

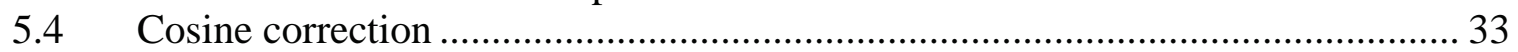

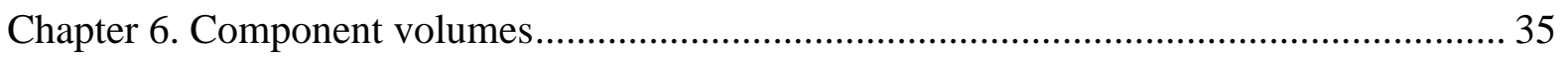

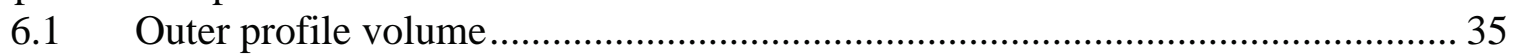

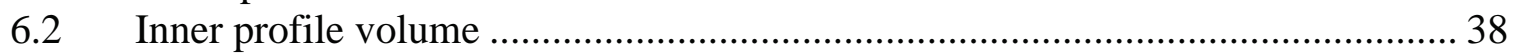

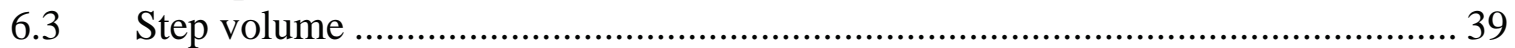

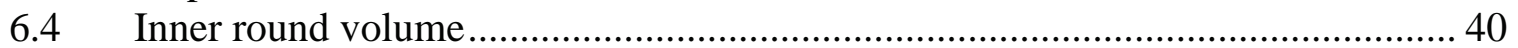

$6.5 \quad$ Outer round volume ................................................................................... 42

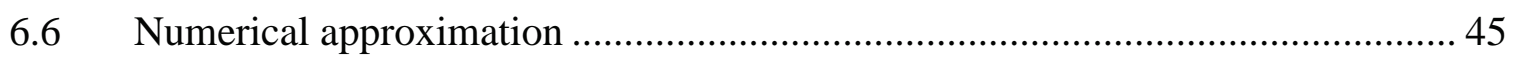

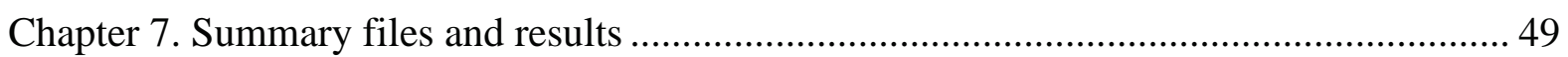

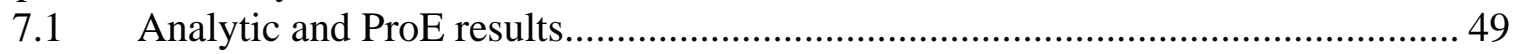

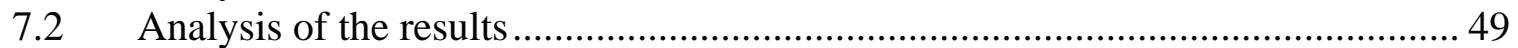

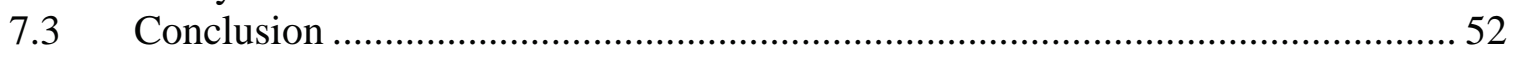

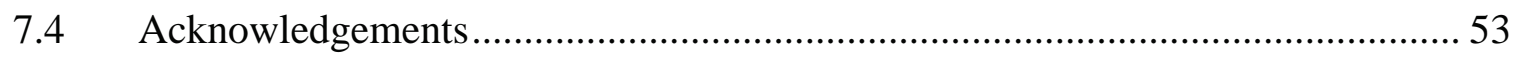

A ppendix A. Drawing of CM M Test A rtifact (157Y -700373) ............................................ 55

A ppendix B. ProE definition at 60 degrees (Scott Schilling) ............................................. 57

A ppendix C. A Iternate analytic solution (Tom B eery) .................................................... 58

A ppendix D. Cosine correction definition (Lowell Savage) ............................................... 61 
Analytic Solutions of an Unclassified Artifact 


\begin{abstract}
This report provides the technical detail for analytic solutions for the inner and outer profiles of the unclassified CM M Test A rtifact (LA N L Part Number 157Y -700373, 5/03/2001) in terms of radius and polar angle. Furthermore, analytic solutions are derived for the legacy Sheffield measurement hardware, al so in terms of radius and polar angle, using part coordinates, i.e., relative to the analytic profile solutions obtained.

The purpose of this work is to determine the exact solution for the "cosine correction" term inherent to measurement with the Sheffield hardware. The cosine correction is required in order to interpret the actual measurements taken by the hardware in terms of an actual part definition, or "knot-point spline definition," that typically accompanies a component drawing.

Specifically, there are two portions of the problem: first an analytic solution must be obtained for any point on the part, e.g., given the radii and the straight lines that define the part, it is required to find an exact solution for the inner and outer profile for any arbitrary polar angle. $\mathrm{N}$ ext, the problem of the inspection of this part must be solved, i.e., given an arbitrary sphere (representing the inspection hardware) that comes in contact with the part (inner and outer profiles) at any arbitrary polar angle, it is required to determine the exact location of that intersection. This is trivial for the case of concentric circles. In the present case, how ever, the spherical portion of the profiles is offset from the defined center of the part, making the analysis nontrivial. Here, a simultaneous solution of the part profiles and the sphere was obtained.
\end{abstract}

\title{
Publication Note
}

This report was previously published by the W eapons System Engineering Division as document number W-2-TR-0017U, Revision A. 
Analytic Solutions of an Unclassified Artifact 


\section{Chapter 1. Analytic part definition}

The part is defined in the drawing on A ppendix A. B oth the inner and outer profiles are combinations of a circle and a straight line. The following definitions are invoked:

$R_{i}$ : radius of the circle for the inner profile $(100 \mathrm{~mm})$

$R_{0}$ : radius of the circle for the outer profile (105 $\mathrm{mm}$ )

$\mathrm{x}_{0}$ : offset of the center of the circles to the part center $(10 \mathrm{~mm})$

$x_{i}$ : distance of the inner profile straight-line section for $x<0(13 \mathrm{~mm})$

$x_{d}$ : additional (maximum) negative $x$-distance of the outer profile $(2 \mathrm{~mm})$

angle $e_{i}$ angle of the inner profile straight-line section (zero)

angle $e_{0}$ : angle of the outer profile straight-line section $(-\pi / 12$ radian, or -15 degrees)

$\mathrm{R}_{\theta}, \mathrm{x}_{\theta}, \mathrm{y}_{\theta}:$ profile definition relative to part center

$\mathrm{R}_{\alpha}, \mathrm{x}_{\alpha}, \mathrm{y}_{\alpha}$ : profile definition relative to circle center

Datum $A$ : vertical line at $x=-x_{i}$ (through edge of inner profile)

The "part center" is at $(x, y)=(0,0)$. Whenever "circle" is used in this report, the threedimensional "sphere" is implied. Certain identities are used repetitively, e.g., $\tan \theta$, for clarity.

\section{$1.1 \quad$ Inner profile}

Figure 1-1 shows the analytic description of the inner profile. The part center is defined at $(x, y)$ of $(0,0)$. The center of the circle is at $\left(x_{0}, 0\right)$. The angle $\theta_{1}$ is the angle from the $x$-axis to the point where the tangent to the circle is horizontal, or

$$
\theta_{1}=\pi / 2-\tan ^{-1}\left(x_{0} / R_{\mathrm{i}}\right) .
$$

The points $(x, y)$ are defined for $\left(0<\theta<\theta_{1}\right)$ and $\left(\theta_{1}<\theta<\theta_{2}\right)$, where $\theta_{2}$ is the angle from the $x$-axis to the point at the most negative $x$-value on the inner profile, as shown in Figure 1-1.

$$
\theta_{2}=\pi / 2+\tan ^{-1}\left(x_{i} / R_{\mathrm{i}}\right) .
$$

Since

$$
R_{\theta}=\sqrt{x_{\theta}{ }^{2}+y_{\theta}{ }^{2}} \text {, and } R_{\alpha}=\sqrt{x_{\alpha}{ }^{2}+y_{\alpha}{ }^{2}}=R_{\mathrm{i}}
$$




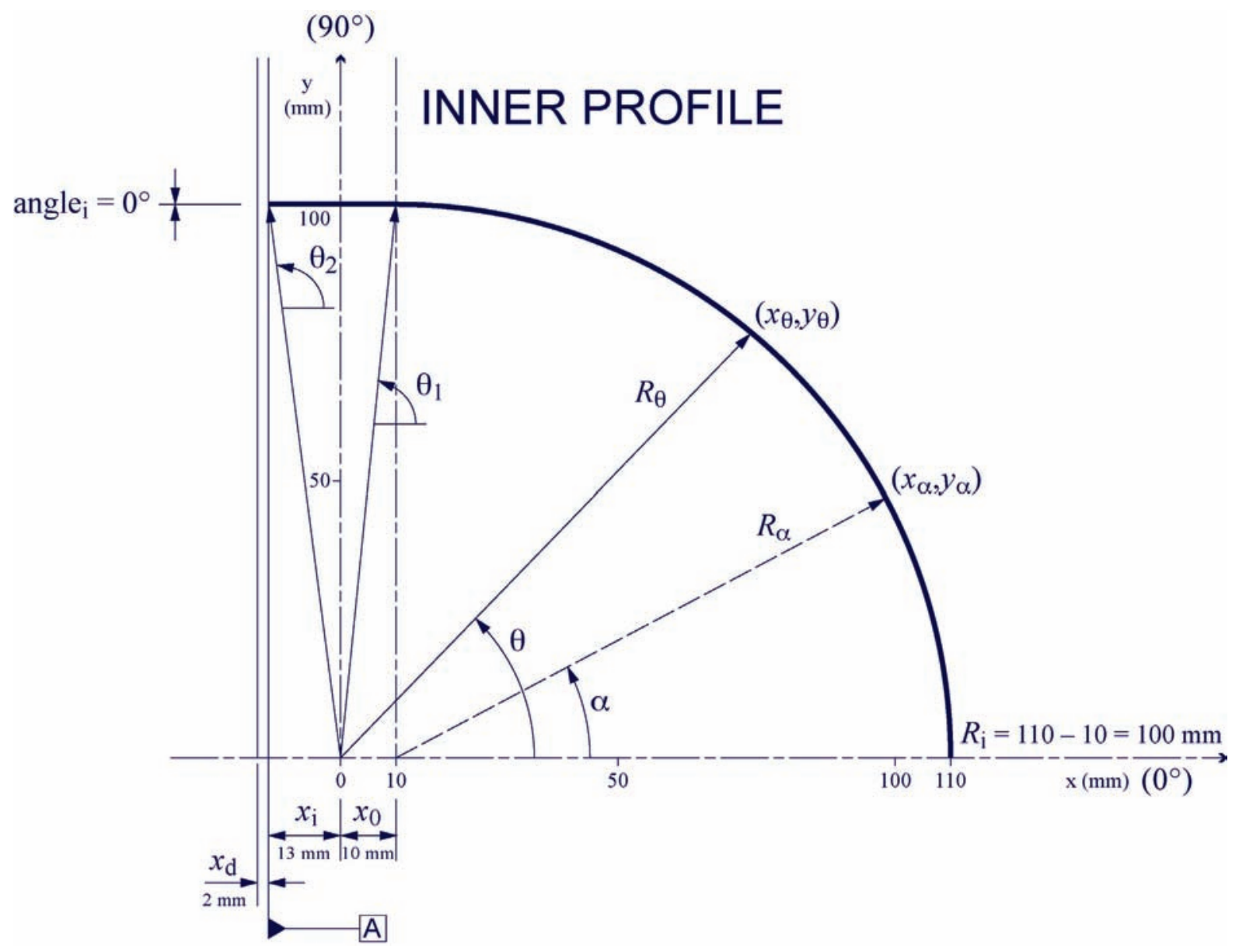

Figure 1-1. Analytic description of inner profile. Datum $A$ is at the edge of the inner profile (not the part center nor the circle center).

and

$$
x_{\alpha}=x_{\theta}-x_{0} \text {, and } y_{\alpha}=y_{\theta} \text {, }
$$

then

$$
R_{\mathrm{i}}=\sqrt{\left(x_{\theta}-x_{0}\right)^{2}+y_{\theta}^{2}} .
$$

Since

$$
\tan \alpha=y_{\theta} /\left(x_{\theta}-x_{0}\right) \text { and } \tan \theta=y_{\theta} / x_{\theta},
$$

the equation for alpha is easily solved as

$$
\alpha=\tan ^{-1}\left[x_{\theta} \tan \theta /\left(x_{\theta}-x_{0}\right)\right],
$$

and 


$$
\theta=\tan ^{-1}\left(\sqrt{\frac{R_{\mathrm{i}}^{2}-\left(x_{\theta}-x_{0}\right)^{2}}{x_{\theta}^{2}}}\right) .
$$

This is an interesting result, but what is required is $\mathrm{x}$ (and $\mathrm{y}$ ) as functions of $\theta$. The solution is straightforward,

$$
x_{\theta}=\frac{x_{0} \pm \sqrt{x_{0}^{2}+\left(1+\tan ^{2} \theta\right)\left(R_{\mathrm{i}}^{2}-x_{0}^{2}\right)}}{1+\tan ^{2} \theta} .
$$

Only the positive root is of interest.

Then

$$
y_{\theta}=x_{\theta} \tan \theta,
$$

and

$$
R_{\theta}=\sqrt{x_{\theta}{ }^{2}+y_{\theta}^{2}}
$$

for $\left(0<\theta<\theta_{1}\right)$.

The straight-line section of the inner profile begins at $\theta_{1}$ and ends at $\theta_{2}$. It is a horizontal line with $y$-intercept at $R_{i}$ (and all values of $y_{\theta}=R_{i}$ ), so

$$
x_{\theta}=R_{\mathrm{i}} / \tan \theta\left(\text { since } x_{\theta} / y_{\theta}=\tan \theta\right),
$$

and

$$
R_{\theta}=\sqrt{x_{\theta}^{2}+y_{\theta}^{2}}
$$

for $\left(\theta_{1}<\theta<\theta_{2}\right)$.

\subsection{Outer profile}

The outer profile is depicted in Figure 1-2. The first step here is to solve for the intersection of the outer profile circle and the straight line. W orking in $\alpha$-space (where the center of the system is the center of the circle),

$$
y_{\alpha}=R_{\mathrm{o}} \sin \alpha \text {, and } x_{\alpha}=R_{\mathrm{o}} \cos \alpha \text {, }
$$




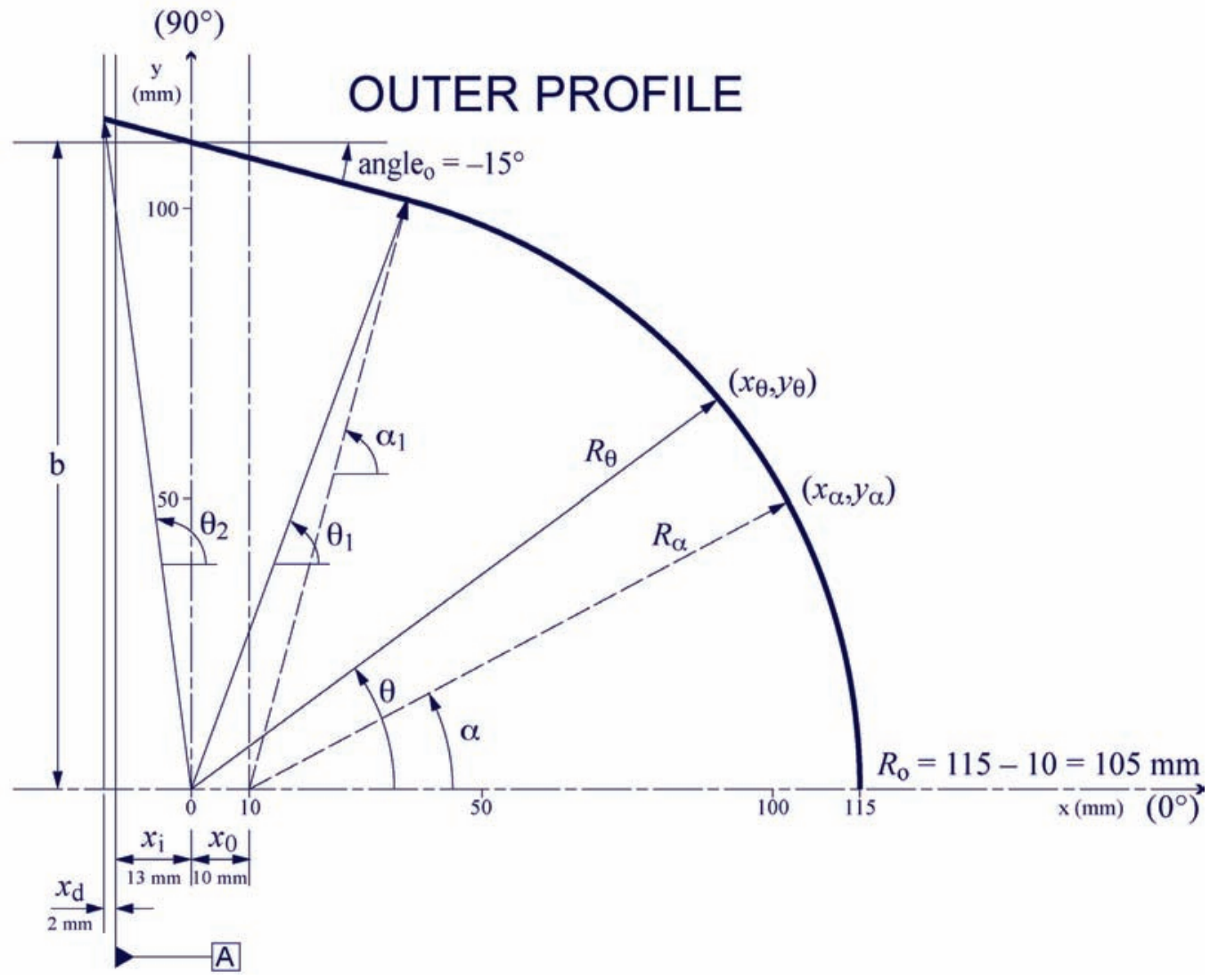

Figure 1-2. Analytic description of the outer profile. Datum $A$ is at the edge of the inner profile (not shown here).

the angle $\alpha_{1}$ is

$$
\alpha_{1}=\pi / 2-\pi / 12
$$

The $x$ and $y$ values at the intersection are therefore

$$
x_{\alpha_{1}}=\sqrt{R_{\mathrm{o}}^{2} /\left(1+\tan ^{2} \alpha_{1}\right)}
$$

using

$$
y_{\alpha_{1}}=x_{\alpha_{1}} \tan \alpha_{1}
$$

Now, the angle through this point in $\theta$-space (the actual center of the part) is

$$
\theta_{1}=\tan ^{-1}\left(\frac{y_{\alpha_{1}}}{x_{\alpha_{1}}+x_{0}}\right)
$$

The equation of the line on the outer profile can be written $y=m x+b$, where 
and

$$
\mathrm{m}=\Delta y / \Delta x
$$

$$
\Delta y=\tan (-\pi / 12) \cdot(-1) \cdot\left(x_{\alpha_{1}}+x_{0}\right)
$$

and the intercept can be solved,

$$
\mathrm{b}=y_{\alpha_{1}}+\Delta y .
$$

It is of interest to solve for the maximum $y$-value on the part since this is given on the drawing in A ppendix A. Here,

$$
\Delta x=x_{0}+x_{i}+x_{d}
$$

and the change in the ordinate (from $y_{\alpha_{1}}$ ) is

$$
\Delta y^{\prime}=-\tan (-\pi / 12) \cdot\left(x_{\alpha_{1}}+10+13+2\right) \approx 13.980517 \mathrm{~mm} .
$$

So the maximum $y$-value on the part is

$$
y_{\text {sharp }}=y_{\alpha_{1}}+\Delta y^{\prime} \approx 115.402729 \mathrm{~mm} \text {. }
$$

Twice this value (to two significant figures) is $230.81 \mathrm{~mm}$, the exact value of the maximum diameter on the drawing in A ppendix A. A dditional detail in the vicinity of the equatorial region of the part is given in Figure 1-3. Note that $x_{\alpha 1}+x_{0}+x_{i}+x_{d}=52.176 \mathrm{~mm}$, identical (to two decimal places) to the drawing tangency point in A ppendix $A$.

The general solution for the $(x, y)$ pairs on the circular portion of the outer profile is determined from the following. These variables are known: $\mathrm{R}_{0}=105 \mathrm{~mm}, \mathrm{x}_{0}=10 \mathrm{~mm}$, and $\alpha_{1}$ $=\pi / 2-\pi / 12$. The following relationships have been previously defined: $x_{\alpha_{1}}$ (Eq. 1-16), $y_{\alpha_{1}}$ (Eq. 1-17), and $\theta_{1}$ (Eq. 1-18). For the region $\left(0<\theta<\theta_{1}\right)$, it is known by definition that

$$
\left(x_{\theta}-x_{0}\right)^{2}+y_{\theta}^{2}=R_{\mathrm{o}}^{2}
$$

and

$$
y_{\theta}=x_{\theta} \tan \theta \text {. }
$$

These two equations can be combined into a quadratic equation and solved for $\mathrm{x}_{\theta}$ :

$$
x_{\theta}=\frac{x_{0} \pm \sqrt{x_{0}^{2}+\left(1+\tan ^{2} \theta\right)\left(R_{\mathrm{o}}^{2}-x_{0}^{2}\right)}}{1+\tan ^{2} \theta} .
$$




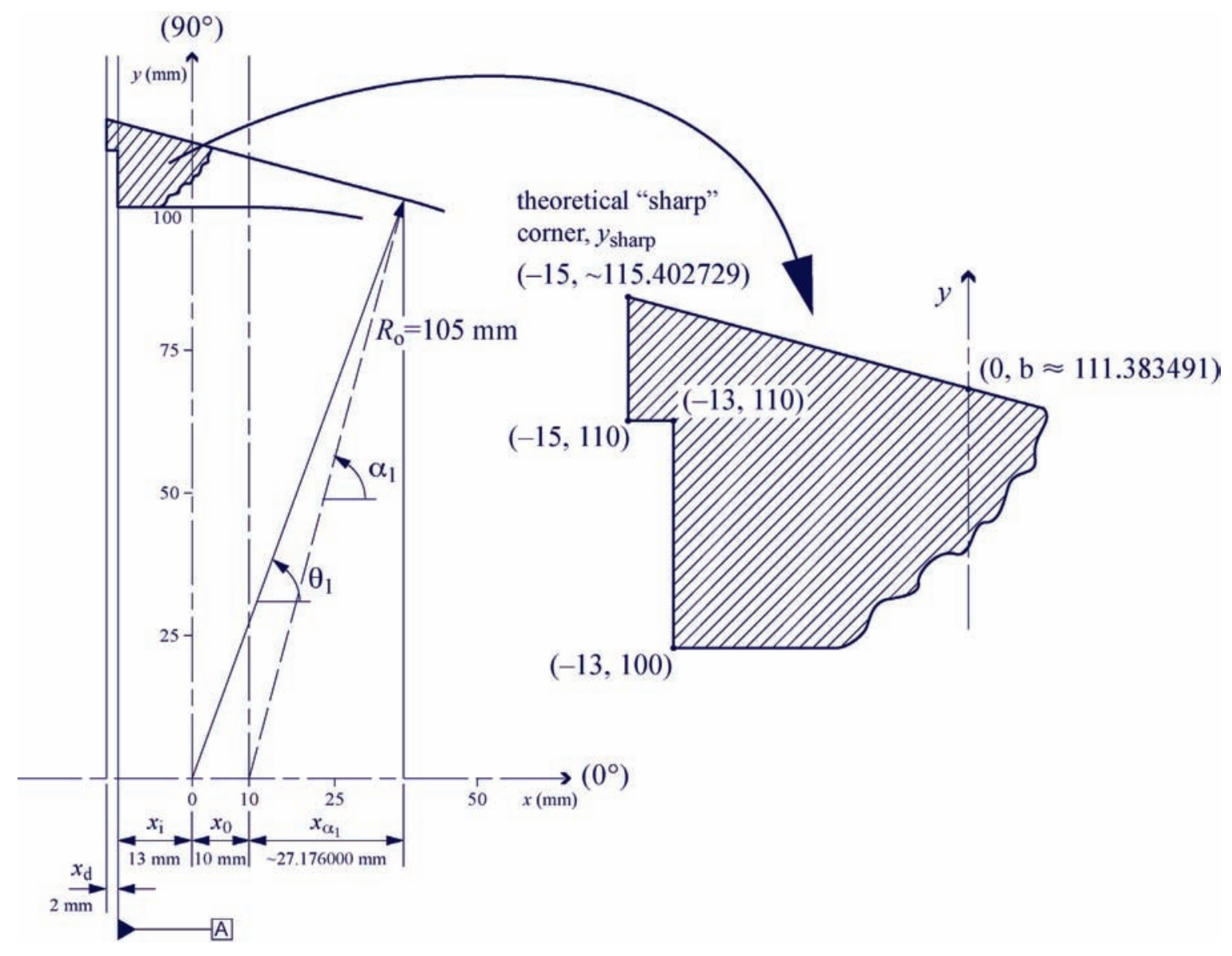

Figure 1-3. Close up of the step region in the outer profile. Note that $x_{\alpha_{1}}+x_{0}+x_{i}+x_{d}=52.176 \mathrm{~mm}$, identical (to two decimal places) to the drawing tangency point in Appendix $A$.

Then $\mathrm{y}_{\theta}$ and $\mathrm{R}_{0}$ can be solved from Eqs. 1-26 and 1-25, respectively.

For $\left(\theta_{1}<\theta<\theta_{2}\right)$, i.e., the straight-line section of the outer profile, the slope can be expressed as the negative reciprocal of $\alpha_{1}$ :

$$
\mathrm{m}=\frac{-1}{\tan (\pi / 2-\pi / 12)}
$$

(N ote: $\theta_{2}$ for the outer profile is solved later.)

Since in the straight line region

$$
y_{\theta}=\mathrm{m} x_{\theta}+\mathrm{b}
$$

and the intercept, $b$, is given by Eq. 1-21, it follows that 


$$
y_{\theta}=\frac{\mathrm{b}}{1-\mathrm{m} / \tan \theta},
$$

and $x_{\theta}$ and $R_{0}$ can be solved from Eqs. 1-26 and 1-25, respectively.

Using these equations, the inner and outer profiles are defined exactly. Figure 1-4 shows the profile contours in $x$-y space and the radius of each profile as a function of angle. Figure 1-5 shows the radial thickness.
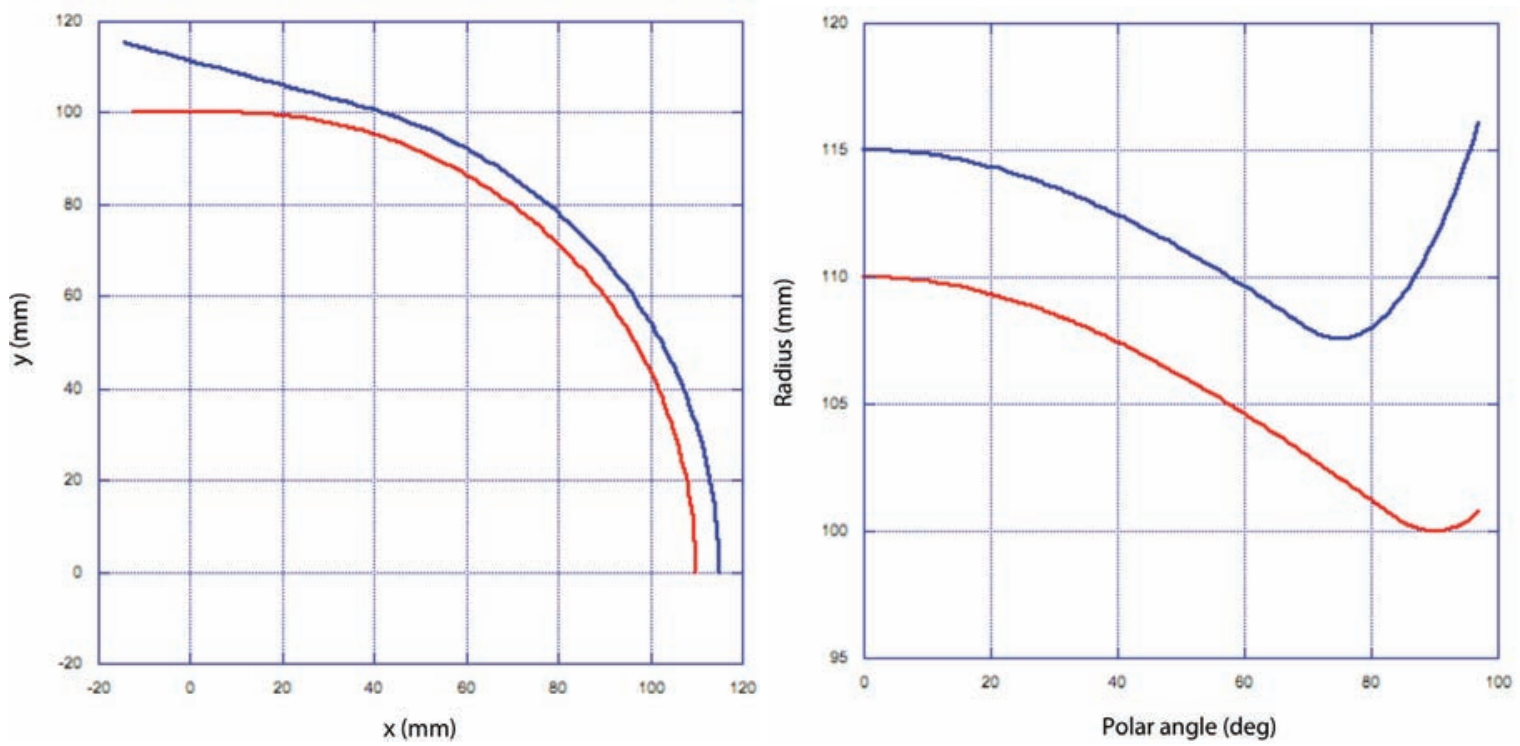

Figure 1-4. $x-y$ profiles (left) and radii vs. $\theta$ (right): inner (red), outer (blue).

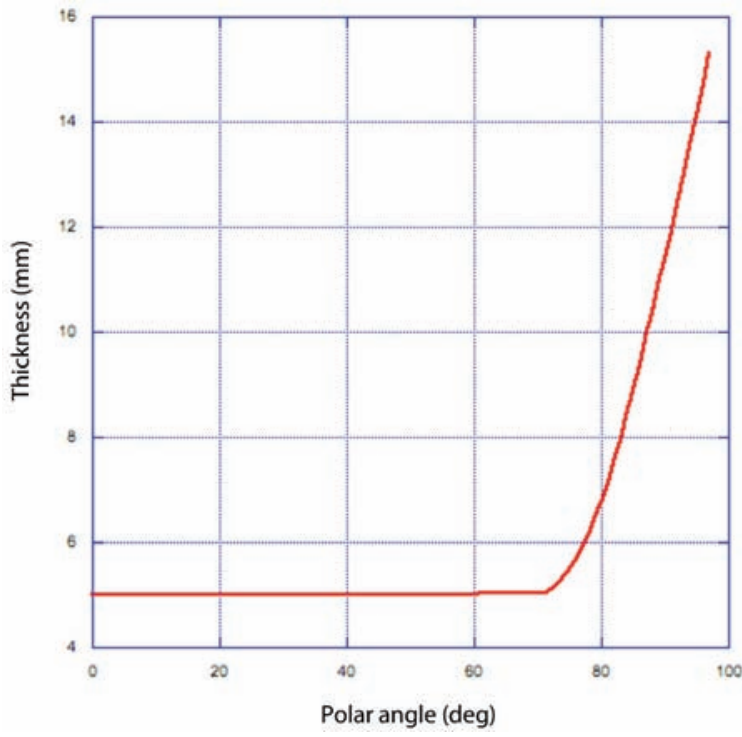

Figure 1-5. Radial thickness (outer less inner) vs. $\theta$. 
Analytic Solutions of an Unclassified Artifact 


\section{Chapter 2. Definition of the inspection sphere}

The inspection of the inner surface of the component is depicted in Figure 2-1. Here, a small sphere of finite radius, representing the inspection hardware, contacts the profile when the sphere is at an arbitrary point $(\theta)$. The size of the sphere in the figure is greatly exaggerated with respect to the component to show details. The following conventions are adopted:

$x_{c}, y_{c}, R_{c}, \theta_{c}$ : the $x, y$, radius, and theta components of the sphere center taken from an origin at the part center

$x_{c c}, y_{c c}, R_{c c}, \theta_{c c}$ : the $x, y$, radius, and theta components of the intersection of the sphere with the profile, i.e., the contact point, taken from an origin at the part center

$\theta$ : the spline angle

$\mathrm{x}_{\theta}, \mathrm{y}_{\theta 1}, \mathrm{R}_{\theta}$ : the $\mathrm{x}, \mathrm{y}$, and radius on the profile at angle $\theta$ (the spline point)

$\left(\mathrm{R}_{\mathrm{cc}}\right)_{\theta}$ : the radius to the sphere edge along the spline angle, $\theta$

$\mathrm{c}=\left(\mathrm{R}_{\mathrm{cc}}\right)_{\theta}-\mathrm{R}_{\theta}$ : the profile "cosine correction" at spline angle, $\theta$

$\phi$ : the diameter of the inspection sphere

The "cosine correction" is the value that must be added to the outer radius of the inspection sphere to determine a radius that would have been measured on the part (if it were possible) at the spline angle, $\theta$. This corrected radius is compared to the theoretical radius at that angle to determine the part deviation of the component (the "as-built" radius) and thereby determine if the part has been manufactured within tolerance. This is required because, as noted, contact does not occur at the polar angle where a measurement is desired, i.e., the spline angle.

The essential fact to be observed in Fig. 2-1 is that the center of the part and the center of the part's spherical contour are not coincident. Therefore, the center of the part, the center of the inspection sphere, and the contact point (of the inspection sphere and the part) are not

collinear, except at $\theta=$ zero. A lso, note that if two circles have a single point of tangency, the two centers and the tangency point are collinear.

\section{$2.1 \quad$ Inner profile}

The entities in Chapter 1 are also applied here, e.g., $\mathrm{R}_{\mathrm{i}}, \theta_{1}$. For any angle $\theta$,

$$
R_{\mathrm{c}}=R_{\theta}-(\phi / 2+c),
$$




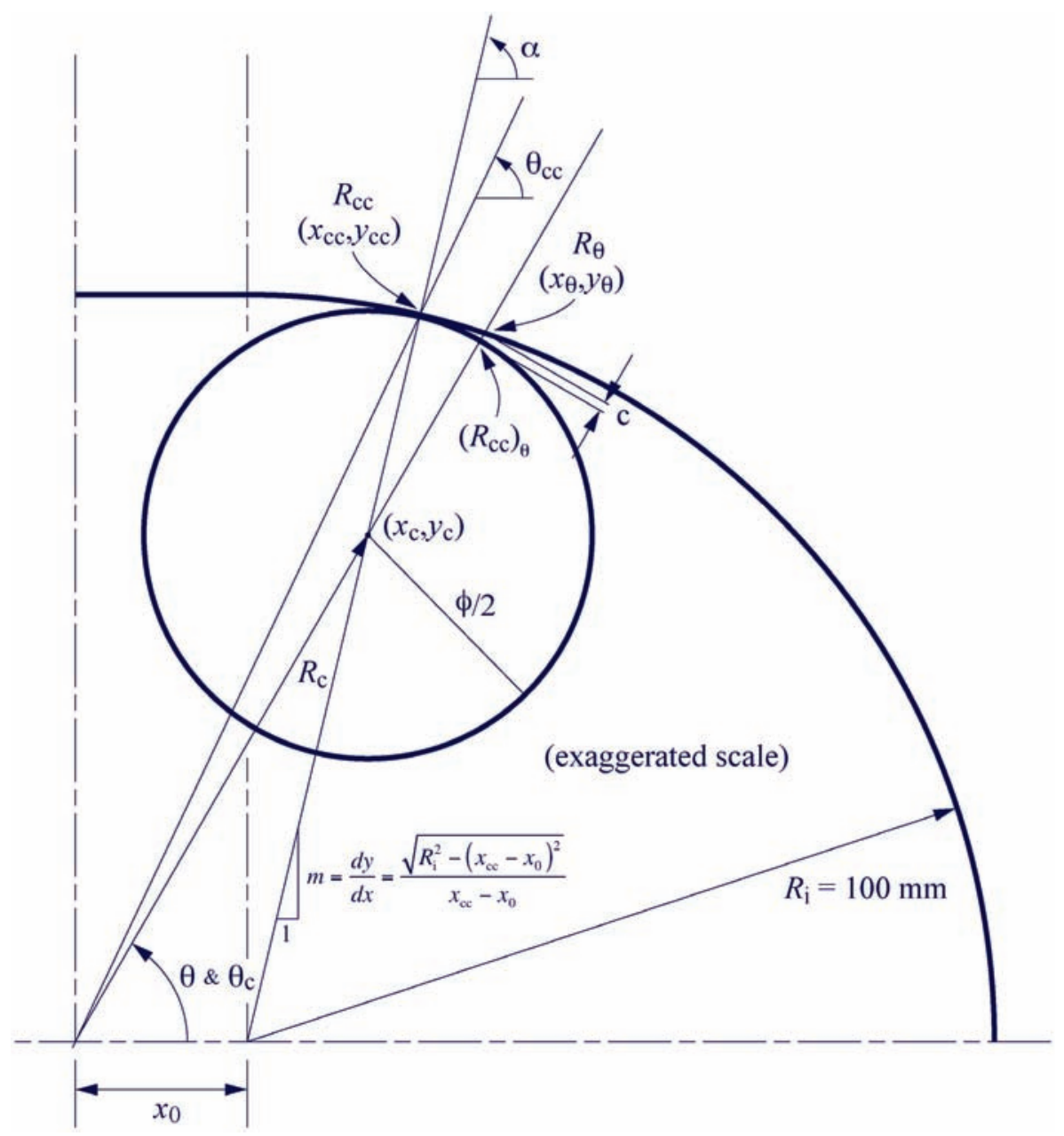

Figure 2-1. Details of the intersection of the inspection sphere (exaggerated scale) and the inner profile of the part.

$$
x_{\mathrm{c}}^{2}+y_{\mathrm{c}}^{2}=R_{\mathrm{c}}^{2}
$$

In the part coordinate system,

$$
\begin{aligned}
& x_{\mathrm{c}}=R_{\mathrm{c}} \cos \theta, \\
& y_{\mathrm{c}}=R_{\mathrm{c}} \sin \theta,
\end{aligned}
$$

so

$$
y_{\mathrm{c}}^{2}=R_{\mathrm{c}}^{2} \sin ^{2} \theta
$$

N ow the inspection sphere (small circle) may be written as:

$$
\left(x-x_{\mathrm{c}}\right)^{2}+\left(y-y_{\mathrm{c}}\right)^{2}=(\phi / 2)^{2} \text {, }
$$


and the large circle, for $0<\theta<\theta_{1}$, is

$$
\left(x-x_{0}\right)^{2}+y^{2}=R_{\mathrm{i}}^{2}
$$

These equations are solved simultaneously for the contact point, $\left(x_{c c}, y_{c c}\right)$, or alternatively, $\left(R_{c c}, \theta_{c c}\right)$, in Chapter 3. This a fundamental point. The link between the small circle and the large circle is only at the contact point. The center of the inspection circle, $\left(x_{c}, y_{c}\right)$, is determined from the geometry only after the solution of the contact point has been accomplished.

\section{$2.2 \quad$ Outer profile}

The intersection of the inspection sphere and the outer spherical surface is shown in Figure 22 . All the conventions used earlier apply here. Note that in this case $\left(R_{c c}\right)_{\theta}>R_{\theta}$, so the cosine correction term will have the opposite sign of that for the inner surface, where $\left(R_{c c}\right)_{\theta}<R_{\theta}$.

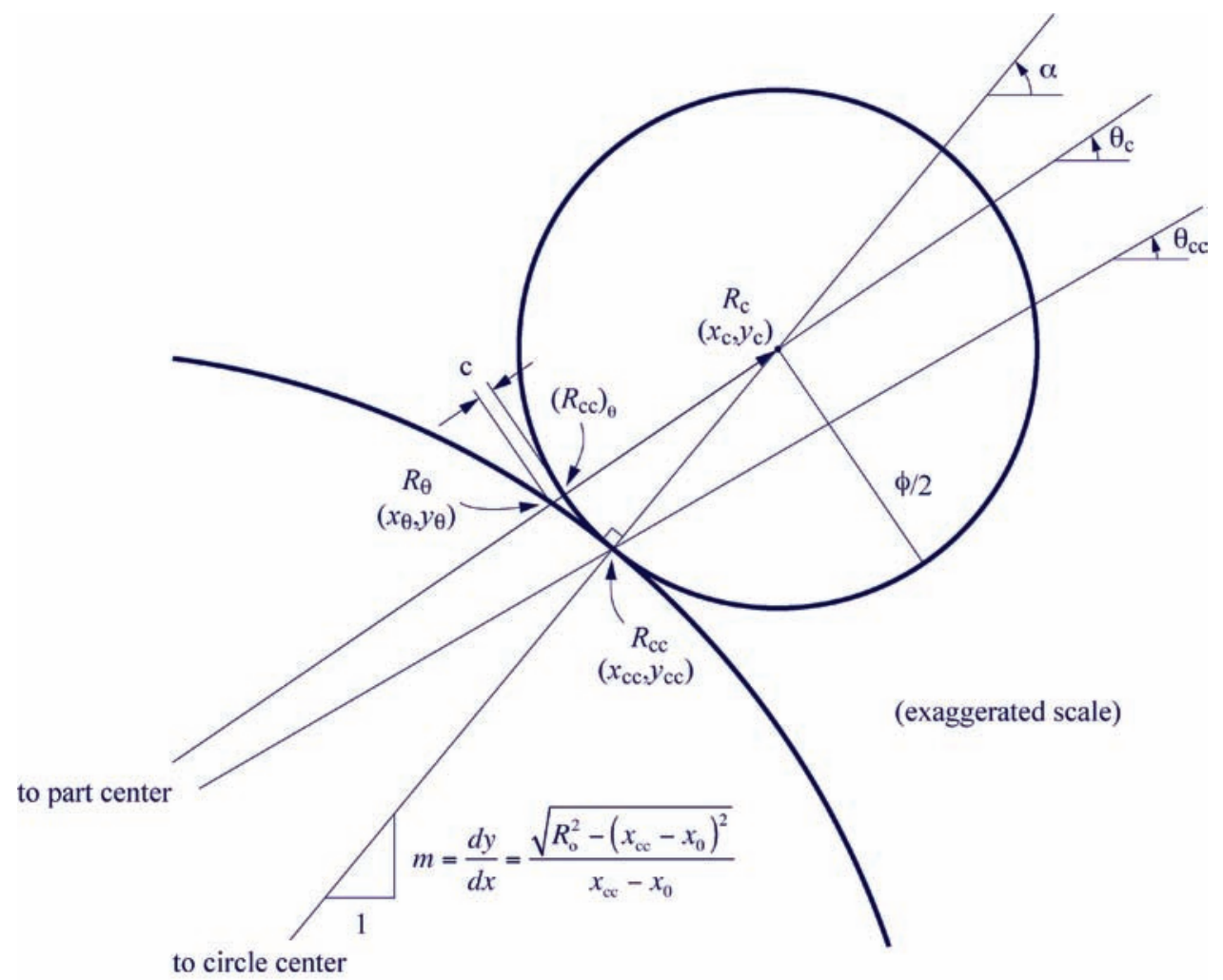

Figure 2-2. Details of the intersection of the inspection sphere (exaggerated scale) and the outer profile of the part.

For any angle $\theta$,

$$
R_{\mathrm{c}}=R_{\theta}+(\phi / 2+c) .
$$


Analytic Solutions of an Unclassified Artifact

Equations 2-2 through 2-5 apply to the outer profile. The inspection sphere (small circle) is written as

$$
\left(x-x_{\mathrm{c}}\right)^{2}+\left(y-y_{\mathrm{c}}\right)^{2}=(\phi / 2)^{2},
$$

and the large circle, for $0<\theta<\theta_{1}$, is

$$
\left(x-x_{\mathrm{o}}\right)^{2}+y^{2}=R_{\mathrm{o}}^{2} .
$$

These final two equations are solved simultaneously for the contact point $\left(x_{c c}, y_{c c}\right)$ on the outer profile at the end of Chapter 3. 


\section{Chapter 3. Simultaneous equations for the contact point on the circular sections}

The purpose of this chapter is to solve Eqs. 2-6 and 2-7 simultaneously to obtain a single equation with a single unknown so that $\left(\mathrm{y}_{\mathrm{cc}}, \mathrm{x}_{\mathrm{cc}}\right)$ can be determined analytically.

As a summary of the problem so far, the following entities are in play:

K nown: $R_{i}, R_{0}, x_{0}, \phi, \theta_{c}$ (the spline point angle, e.g., $\pi / 3$ )

$N$ ot relevant for the solution: $R_{\theta}$ (the spline point radius)

Unknown: $R_{c c}, \theta_{c c}\left(x_{c c}, y_{c c}\right)$, and $R_{c}$

Equations: probe sphere (Eq. 2-6) and inner profile (Eq. 2-7)

A gain, the goal is to solve for the contact point, $\left(x_{c c}, y_{c c}\right)$.

\subsection{Inner profile}

The two equations at the contact point are, for the large circle, for $0<\theta<\theta_{1}$,

$$
\left(x_{\mathrm{cc}}-x_{0}\right)^{2}+y_{\mathrm{cc}}{ }^{2}=R_{\mathrm{i}}^{2},
$$

and the inspection sphere (small circle) is

$$
\left(x_{\mathrm{cc}}-x_{\mathrm{c}}\right)^{2}+\left(y_{\mathrm{cc}}-y_{\mathrm{c}}\right)^{2}=\phi^{2} / 4 .
$$

The slope through the contact point $\left(x_{c c}, y_{c c}\right)$ and the probe sphere center $\left(x_{c}, y_{c}\right)$ is $-1 /(d y / d x)$ of the profile, or

$$
\mathrm{m}=\frac{\sqrt{R_{\mathrm{i}}^{2}-\left(x-x_{0}\right)^{2}}}{x-x_{0}} .
$$

When $\mathrm{x}=\mathrm{x}_{\mathrm{cc}}$,

$$
\mathrm{m}=\frac{\sqrt{R_{\mathrm{i}}^{2}-\left(x_{\mathrm{cc}}-x_{0}\right)^{2}}}{x_{\mathrm{cc}}-x_{0}} .
$$

By the definition of the slope,

$$
\mathrm{m}=\left(y_{\mathrm{cc}}-y_{\mathrm{c}}\right) /\left(x_{\mathrm{cc}}-x_{\mathrm{c}}\right),
$$


so

$$
\left(y_{\mathrm{cc}}-y_{\mathrm{c}}\right)^{2}=\mathrm{m}^{2}\left(x_{\mathrm{cc}}-x_{\mathrm{c}}\right)^{2} \text {. }
$$

Therefore from Eq. 3-2,

$$
\begin{aligned}
& \left(x_{\mathrm{cc}}-x_{\mathrm{c}}\right)^{2}\left(1+\mathrm{m}^{2}\right)=\phi^{2} / 4 \\
& x_{\mathrm{c}}=x_{\mathrm{cc}}-\frac{\phi / 2}{\sqrt{1+\mathrm{m}^{2}}} .
\end{aligned}
$$

Also, from Eq. 3-5

$$
y_{\mathrm{c}}=y_{\mathrm{cc}}-\mathrm{m}\left(x_{\mathrm{cc}}-x_{\mathrm{c}}\right) \text {. }
$$

Now, it is also known, by definition, that

$$
\theta_{\mathrm{c}}=\tan ^{-1}\left(y_{\mathrm{c}} / x_{\mathrm{c}}\right)
$$

then

$$
y_{\mathrm{c}}=x_{\mathrm{c}} \tan \theta_{\mathrm{c}} \quad\left(\text { so } x_{\mathrm{c}}=y_{\mathrm{c}} / \tan \theta_{\mathrm{c}}\right) .
$$

From Eq. 3-1,

$$
y_{\mathrm{cc}}=\sqrt{R_{\mathrm{i}}^{2}-\left(x_{\mathrm{cc}}-x_{0}\right)^{2}},
$$

and from Eq. 3-9,

$$
y_{\mathrm{cc}}=y_{\mathrm{c}}+\mathrm{m}\left(x_{\mathrm{cc}}-x_{\mathrm{c}}\right) \text {. }
$$

Therefore,

$$
\sqrt{R_{\mathrm{i}}^{2}-\left(x_{\mathrm{cc}}-x_{0}\right)^{2}}=y_{\mathrm{c}}+\mathrm{m}\left(x_{\mathrm{cc}}-x_{\mathrm{c}}\right) \text {. }
$$

Now substituting Eq. 3-11 for $\mathrm{y}_{\mathrm{c}}$

$$
\sqrt{R_{\mathrm{i}}^{2}-\left(x_{\mathrm{cc}}-x_{0}\right)^{2}}=x_{\mathrm{c}} \tan \theta_{\mathrm{c}}+\mathrm{m}\left(x_{\mathrm{cc}}-x_{\mathrm{c}}\right) \text {, }
$$

or

$$
\sqrt{R_{\mathrm{i}}^{2}-\left(x_{\mathrm{cc}}-x_{0}\right)^{2}}=x_{\mathrm{c}}\left(\tan \theta_{\mathrm{c}}-\mathrm{m}\right)+\mathrm{m} x_{\mathrm{cc}}
$$


or

$$
x_{\mathrm{c}}=\frac{\sqrt{R_{\mathrm{i}}^{2}-\left(x_{\mathrm{cc}}-x_{0}\right)^{2}}-\mathrm{m} x_{\mathrm{cc}}}{\left(\tan \theta_{\mathrm{c}}-\mathrm{m}\right)} .
$$

Finally, substituting for $x_{c}$ from Eq. 3-8,

$$
x_{\mathrm{cc}}-\frac{\phi / 2}{\sqrt{1+\mathrm{m}^{2}}}=\frac{\sqrt{R_{\mathrm{i}}^{2}-\left(x_{\mathrm{cc}}-x_{0}\right)^{2}}-\mathrm{m} x_{\mathrm{cc}}}{\left(\tan \theta_{\mathrm{c}}-\mathrm{m}\right)} .
$$

So here we have a single equation with a single unknown, $x_{c c}$. Rewriting this, and using Eq. 3-4 for m, Eq. 3-19 will be solved in Chapter 4 using the Newton-Raphson method.

$$
\begin{gathered}
x_{\mathrm{cc}}-\frac{\phi / 2}{\sqrt{1+\left(\frac{\sqrt{R_{\mathrm{i}}^{2}-\left(x_{\mathrm{cc}}-x_{0}\right)^{2}}}{\left(x_{\mathrm{cc}}-x_{0}\right)}\right)^{2}}} \\
-\frac{\sqrt{R_{\mathrm{i}}^{2}-\left(x_{\mathrm{cc}}-x_{0}\right)^{2}}-x_{\mathrm{cc}} \frac{\sqrt{R_{\mathrm{i}}^{2}-\left(x_{\mathrm{cc}}-x_{0}\right)^{2}}}{\left(x_{\mathrm{cc}}-x_{0}\right)}}{\tan \theta_{\mathrm{c}}-\frac{\sqrt{R_{\mathrm{i}}^{2}-\left(x_{\mathrm{cc}}-x_{0}\right)^{2}}}{\left(x_{\mathrm{cc}}-x_{0}\right)}}=0
\end{gathered}
$$

\subsection{Outer profile}

This analysis follows the inner profile equations, with a few important differences. The equation at the contact point for the large circle for $0<\theta<\theta_{1}$ is

$$
\left(x_{\mathrm{cc}}-x_{0}\right)^{2}+y^{2}=R_{\mathrm{o}}^{2},
$$

and the equation for the inspection sphere (small circle) is

$$
\left(x_{\mathrm{cc}}-x_{\mathrm{c}}\right)^{2}+\left(y_{\mathrm{cc}}-y_{\mathrm{c}}\right)^{2}=(\phi / 2)^{2} \text {. }
$$

From Eq. 3-20, the slope on the outer profile is

$$
\frac{\mathrm{d} y}{\mathrm{~d} x}=\frac{x_{0}-x}{\sqrt{R_{\mathrm{o}}^{2}-\left(x-x_{0}\right)^{2}}} .
$$


The slope of the line from the probe center $\left(x_{c}, y_{c}\right)$ to the contact point $\left(x_{c c}, y_{c c}\right)$ is, by definition, the negative reciprocal of the slope, or at $x=x_{c c}$,

$$
\mathrm{m}=\frac{-1}{(\mathrm{~d} y / \mathrm{d} x)}=\frac{\sqrt{R_{\mathrm{o}}^{2}-\left(x_{\mathrm{cc}}-x_{0}\right)^{2}}}{x_{\mathrm{cc}}-x_{0}} .
$$

By definition, the slope

$$
\mathrm{m} \equiv \frac{\Delta y}{\Delta x}=\frac{y_{\mathrm{c}}-y_{\mathrm{cc}}}{x_{\mathrm{c}}-x_{\mathrm{cc}}}
$$

so

$$
\left(y_{\mathrm{cc}}-y_{\mathrm{c}}\right)^{2}=\mathrm{m}^{2}\left(x_{\mathrm{cc}}-x_{\mathrm{c}}\right)^{2} .
$$

By substitution of Eq. 3-25 into Eq. 3-21, and by choosing the positive root (different from the solution of the inner profile),

$$
x_{\mathrm{c}}=x_{\mathrm{cc}}+\frac{\phi / 2}{\sqrt{1+\mathrm{m}^{2}}} .
$$

Also, from Eq. 3-24,

$$
y_{\mathrm{c}}=y_{\mathrm{cc}}+\mathrm{m}\left(x_{\mathrm{c}}-x_{\mathrm{cc}}\right)
$$

Note the sign change from the inner profile definition given in Eq. 3-8. B oth roots are possible, but in each case, only one is on the profile surface.

Equations 3-10 and 3-11 apply to the outer profile as well, so from Eq. 3-20,

$$
y_{\mathrm{cc}}=\sqrt{R_{\mathrm{o}}^{2}-\left(x_{\mathrm{cc}}-x_{0}\right)^{2}},
$$

and from Eq. 3-27,

$$
y_{\mathrm{cc}}=y_{\mathrm{c}}-\mathrm{m}\left(x_{\mathrm{c}}-x_{\mathrm{cc}}\right) \text {. }
$$

Therefore,

$$
\sqrt{R_{\mathrm{o}}^{2}-\left(x_{\mathrm{cc}}-x_{0}\right)^{2}}=y_{\mathrm{c}}-\mathrm{m}\left(x_{\mathrm{c}}-x_{\mathrm{cc}}\right),
$$

and substituting for $\mathrm{y}_{\mathrm{c}}$ from Eq. 3-11, 


$$
\sqrt{R_{\mathrm{o}}^{2}-\left(x_{\mathrm{cc}}-x_{0}\right)^{2}}=x_{\mathrm{c}} \tan \theta_{\mathrm{c}}-\mathrm{m}\left(x_{\mathrm{c}}-x_{\mathrm{cc}}\right) .
$$

Finally, solving for $x_{c}$

$$
x_{\mathrm{c}}=\frac{\sqrt{R_{\mathrm{o}}^{2}-\left(x_{\mathrm{cc}}-x_{0}\right)^{2}}-\mathrm{m} x_{\mathrm{cc}}}{\tan \theta_{\mathrm{c}}-\mathrm{m}},
$$

and using the definition of $x_{c}$ in Eq. 3-26,

$$
x_{\mathrm{cc}}+\frac{\phi / 2}{\sqrt{1+\mathrm{m}^{2}}}=\frac{\sqrt{R_{\mathrm{o}}^{2}-\left(x_{\mathrm{cc}}-x_{0}\right)^{2}}-\mathrm{m} x_{\mathrm{cc}}}{\tan \theta_{\mathrm{c}}-\mathrm{m}} .
$$

Rewriting this and using Eq. 3-23 for $\mathrm{m}$,

$$
\begin{gathered}
x_{\mathrm{cc}}+\frac{\phi / 2}{\sqrt{1+\left(\frac{\sqrt{R_{\mathrm{o}}^{2}-\left(x_{\mathrm{cc}}-x_{0}\right)^{2}}}{x_{\mathrm{cc}}-x_{0}}\right)^{2}}} \\
-\frac{\sqrt{R_{\mathrm{o}}^{2}-\left(x_{\mathrm{cc}}-x_{0}\right)^{2}}-x_{\mathrm{cc}} \frac{\sqrt{R_{\mathrm{o}}^{2}-\left(x_{\mathrm{cc}}-x_{0}\right)^{2}}}{x_{\mathrm{cc}}-x_{0}}}{\tan \theta_{\mathrm{c}}-\frac{\sqrt{R_{\mathrm{o}}^{2}-\left(x_{\mathrm{cc}}-x_{0}\right)^{2}}}{x_{\mathrm{cc}}-x_{0}}}=0
\end{gathered}
$$

Notice that except for the sign of the second term and the substitution of $R_{0}$ for $R_{i}$, this equation is the same as Eq. 3-19, for the inner profile. 
Analytic Solutions of an Unclassified Artifact 


\section{Chapter 4. Newton-R aphson solution for the circular sections}

\subsection{Inner circular profile}

The N ewton-R aphson technique allows the rapid numerical finding of zeros of a continuous, differentiable function. In Chapter 3, two simultaneous functions were solved to obtain a single equation with a single unknown variable. Eq. 3-19 shows the equation where the unknown variable is the $x$-component of the contact point, $x_{c c}$. It occurs in nine places in the four-term equation. It may be written as,

$$
\mathrm{F}(x)=\mathrm{f}_{1}(x)-\mathrm{f}_{2}(x)-\mathrm{f}_{3}(x)+\mathrm{f}_{4}(x)=0 .
$$

The derivative may be written as,

$$
\mathrm{F}^{\prime}(x)=\mathrm{f}_{1}^{\prime}(x)-\mathrm{f}_{2}^{\prime}(x)-\mathrm{f}_{3}^{\prime}(x)+\mathrm{f}_{4}^{\prime}(x)=0 .
$$

From Eq. 3-19, the terms in Eq. 4-1 may be written as

$$
\begin{aligned}
& \mathrm{f}_{1}(x)=x, \\
& \mathrm{f}_{2}(x)=\frac{\phi / 2}{\sqrt{1+\frac{\mathrm{q}(x)^{2}}{\mathrm{p}(x)^{2}}}}, \\
& \mathrm{f}_{3}(x)=\frac{\mathrm{q}(x)}{\tan \theta_{c}-\frac{\mathrm{q}(x)}{\mathrm{p}(x)}}, \\
& \mathrm{f}_{4}(x)=\frac{\mathrm{q}(x)}{\mathrm{p}(x)} \\
& \tan \theta_{\mathrm{c}}-\frac{\mathrm{q}(x)}{\mathrm{p}(x)}
\end{aligned}
$$

where

$$
\mathrm{p}(x)=x-x_{0}
$$

and

$$
\mathrm{q}(x)=\sqrt{R_{\mathrm{i}}^{2}-\left(x-x_{0}\right)^{2}} .
$$

Each term, $\mathrm{f}_{1}(\mathrm{x})$ through $\mathrm{f}_{4}(\mathrm{x})$ may be differentiated with multiple application of the product and quotient rules: 


$$
\frac{\mathrm{d}}{\mathrm{d} x}[\mathrm{r}(x) \mathrm{s}(x)]=\mathrm{r}(x) \frac{\mathrm{d}}{\mathrm{d} x} \mathrm{~s}(x)+\mathrm{s}(x) \frac{\mathrm{d}}{\mathrm{d} x} \mathrm{r}(x)
$$

and

$$
\frac{\mathrm{d}}{\mathrm{d} x}\left[\frac{\mathrm{u}(x)}{\mathrm{v}(x)}\right]=\frac{\mathrm{v}(x) \frac{\mathrm{d}}{\mathrm{d} x} \mathrm{u}(x)-\mathrm{u}(x) \frac{\mathrm{d}}{\mathrm{d} x} \mathrm{v}(x)}{\mathrm{v}(x)^{2}}
$$

respectively.

The individual terms of the derivative in Eq. 4-2 are

$$
\begin{aligned}
& \mathrm{f}_{1}^{\prime}(x)=1, \\
& \mathrm{f}_{2}^{\prime}(x)=\frac{\frac{\phi}{2} \frac{R_{\mathrm{i}}^{2}}{\mathrm{p}(x)^{3}}}{\left[1+\frac{\mathrm{q}(x)^{2}}{\mathrm{p}(x)^{2}}\right]^{3 / 2}}, \\
& \mathrm{f}_{3}^{\prime}(x)=\frac{-\tan \theta_{\mathrm{c}} \mathrm{p}(x)}{[\operatorname{q}(x)}-\frac{\mathrm{q}(x)^{2}}{\mathrm{p}(x)^{2}} \\
& \mathrm{f}_{4}^{\prime}(x)=\frac{\left.\tan \theta_{\mathrm{c}}-\frac{\mathrm{q}(x)]^{2}}{\mathrm{p}(x)}\right]^{2}\left[\frac{\mathrm{q}(x)}{\mathrm{p}(x)}-\frac{x}{\mathrm{q}(x)}-\frac{x \mathrm{q}(x)}{\mathrm{p}(x)^{2}}\right]-\frac{\mathrm{q}(x)^{2}}{\mathrm{p}(x)^{2}}}{\left[\tan \theta_{\mathrm{c}}-\frac{\mathrm{q}(x)]^{2}}{\mathrm{p}(x)}\right]^{2}},
\end{aligned}
$$

Given a reasonably close guess, $x_{n}$, to the solution of $E q$. 4-1 (the contact point between the inspection machine sphere and the component profile) then N ewton-Raphson provides a next iterative approximation:

$$
x_{n+1}=x_{n}-\frac{\mathrm{f}\left(x_{n}\right)}{\mathrm{f}^{\prime}\left(x_{n}\right)} \text {. }
$$

The error in this new approximation may be expressed as a percentage:

$$
\operatorname{err}_{n+1}=\left|\frac{x_{n+1}-x_{n}}{x_{n}}\right| \times 100
$$


This solution technique is very simple and converges extremely quickly for a "well-behaved" function. This is true in the present case. Equally important for rapid convergence, an excellent guess for the solution of Eq. 4-1 may be readily obtained from the positive root of the analytic solution of the profile point, $\mathrm{x}_{\theta}$ (at angle $\theta$ ) in Eq. 1-9, repeated here,

$$
x_{\theta}=\frac{x_{0}+\sqrt{x_{0}^{2}+\left(1+\tan ^{2} \theta\right)\left(R_{\mathrm{i}}^{2}-x_{0}^{2}\right)}}{1+\tan ^{2} \theta} .
$$

This is a good choice for a guess, since the actual required solution point, $x_{c c}$, is very close to the spline point $x_{\theta}$, as shown in Figure 2-1. Indeed, the solution of every case (for $\theta<\theta_{1}$ ) converges to an error (Eq. 4-16) of $1.0 \times 10^{-12}$ in seven (or less) steps.

\subsection{Outer circular profile}

The solution for the circular section of the outer contour is nearly the same as for the inner. The substitution of the outer radius for the inner radius is obvious, however, there is a more subtle sign change in term $\mathrm{f}_{2}(\mathrm{x})$ that is required in order to solve for the "right" circle. In fact, the simultaneous solution of the inspection sphere and the circular section of the profiles yields two circles as shown in Figure 4-1. Only one is physical, however, since the inspection sphere must approach the inner profile from the inside, and the outer profile from the outside. The following are taken from Eq. 3-34:

$$
\mathrm{F}(x)=\mathrm{f}_{1}(x)+\mathrm{f}_{2}(x)-\mathrm{f}_{3}(x)+\mathrm{f}_{4}(x)=0,
$$

and the derivative is therefore,

$$
\mathrm{F}^{\prime}(x)=\mathrm{f}_{1}^{\prime}(x)+\mathrm{f}_{2}^{\prime}(x)-\mathrm{f}_{3}^{\prime}(x)+\mathrm{f}_{4}^{\prime}(x)=0 .
$$

The terms of the function and the derivative for this case of the outer profile are identical to those in Eqs. 4-3 through 4-6 and Eqs. 4-11 through 4-14.

The function $q(x)$, however, is defined in terms of the outer profile radius,

$$
\mathrm{q}(x)=\sqrt{R_{\mathrm{o}}^{2}-\left(x-x_{0}\right)^{2}} .
$$

Figures 4-2 and 4-3 illustrate the function of the contact point $F\left(x=x_{c c}\right)$ and the derivative for the inner and outer profiles, respectively. 


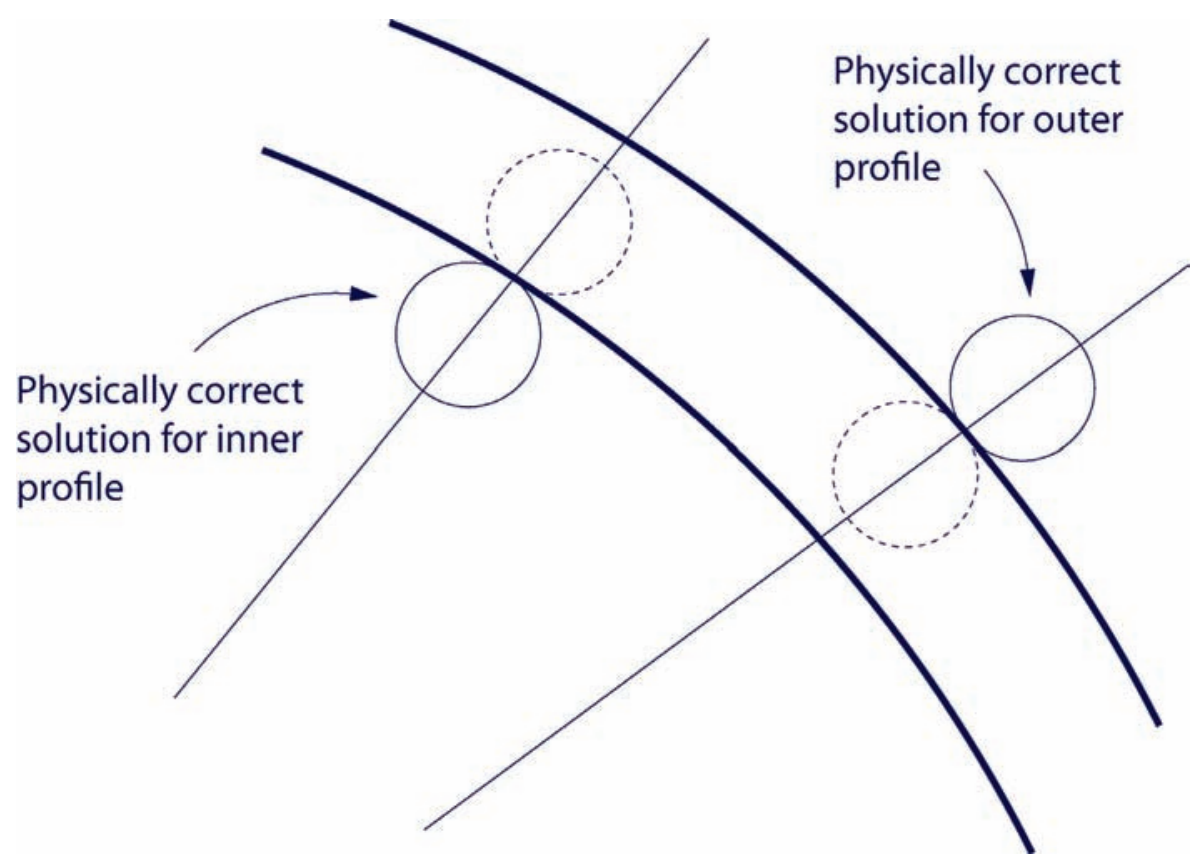

Figure 4-1. Illustration of the two circles that intersect the inner and outer profiles. In each case, only one circle is physically possible.

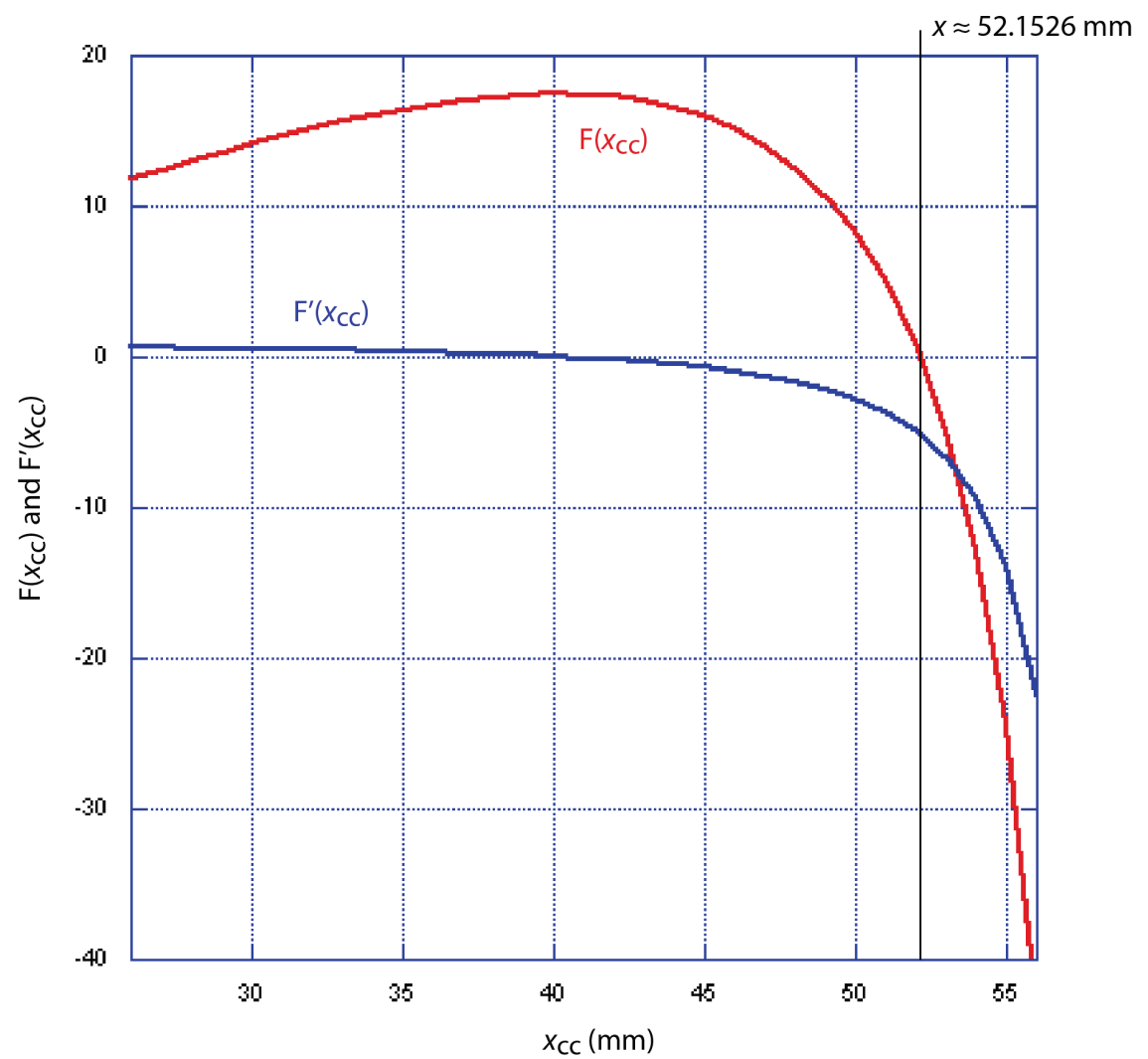

Figure 4-2. Plot for the inner profile at $\theta=60$ degrees of the function $\mathrm{F}(x)$ and its derivative, $\mathrm{F}^{\prime}(x)$, defined in Eqs. 4-1 and 4-2, respectively. The zero of $F(x)$ is shown graphically at approximately $52.1526 \mathrm{~mm}$. 


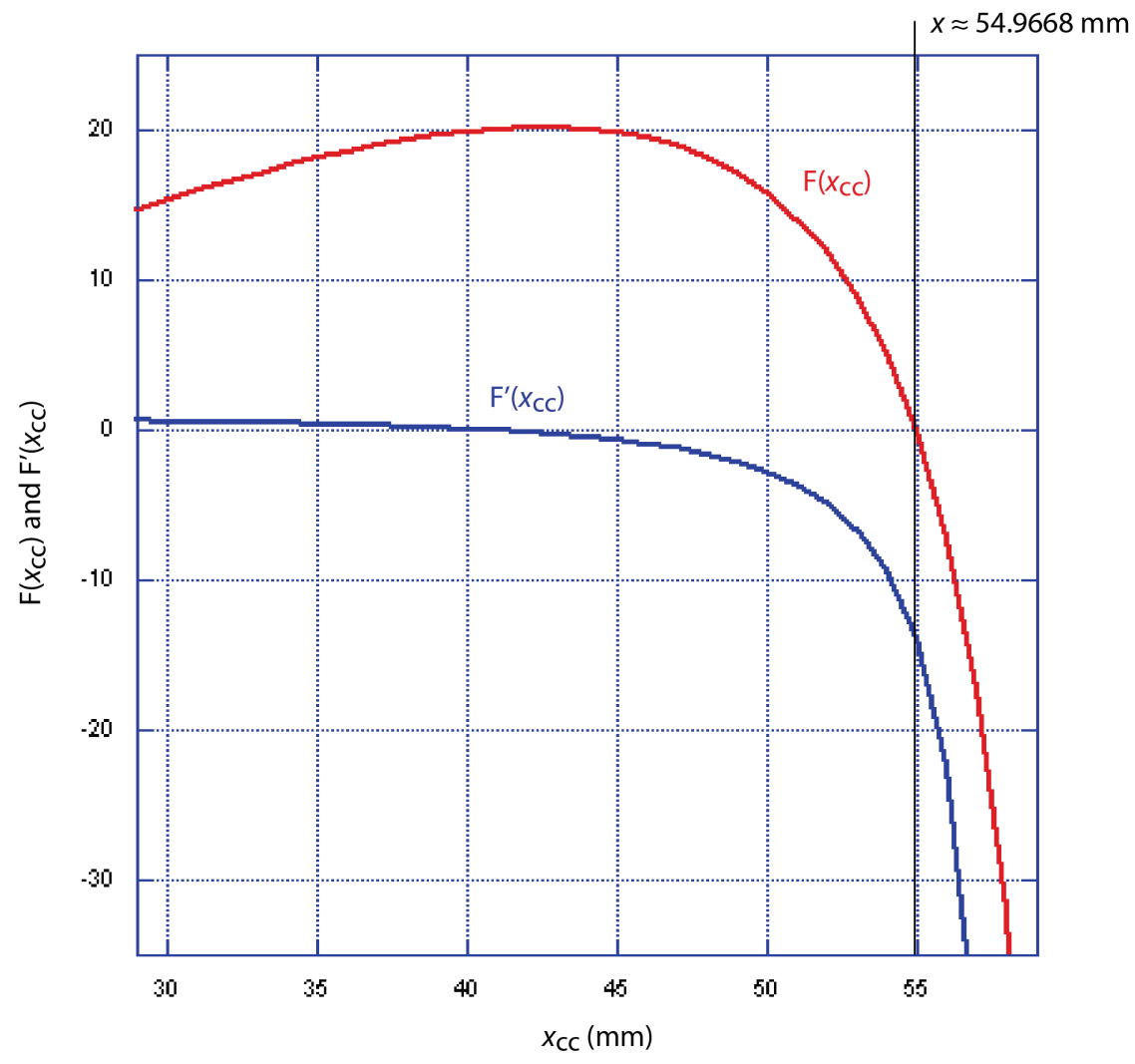

Figure 4-3. Plot for the outer profile at $\theta=60$ degrees of the function $\mathrm{F}(x)$ and its derivative, $\mathrm{F}^{\prime}(x)$, defined in Eqs. 4-18 and 4-19, respectively. The zero of $F(x)$ is shown graphically at approximately $54.9668 \mathrm{~mm}$. 
Analytic Solutions of an Unclassified Artifact 


\section{Chapter 5. Solutions for the linear sections}

The linear solution is similar to the setup for the circular section solutions. It is simpler and combines the contact definition (as in Chapter 2) and the solution (as in Chapter 3). Since the solution is much simpler for the case of the intersection of a line and a circle, it can be solved analytically without the need of a numerical solver (as in Chapter 4).

\subsection{Inner linear profile}

As in Chapter 3, the known quantities for this problem are

$\mathrm{R}_{\mathrm{i}}, \mathrm{x}_{0}, \phi$, and $\theta_{\mathrm{c}}$ (the spline point angle, e.g., $\pi / 3$ ),

and the unknowns are

$$
\mathrm{R}_{\mathrm{cc},}, \theta_{\mathrm{cc}}\left(\mathrm{x}_{\mathrm{cc},} \mathrm{Y}_{\mathrm{cc}}\right) \text {, and } \mathrm{R}_{\mathrm{c}} \text {. }
$$

The intersection of the inspection sphere and the horizontal line that defines the inner profile for angles is illustrated in Figure 5-1, which is similar to Figure 2-1, except that the inner profile is linear. Figure 5-1 shows the intersection at a general spline angle for $\theta_{c}<\theta_{c c}$ and for $\theta_{c}>\theta_{c c}$, like, for example, the final point on the inner surface. A $s$ it turns out, the solution is general and applies for both cases.

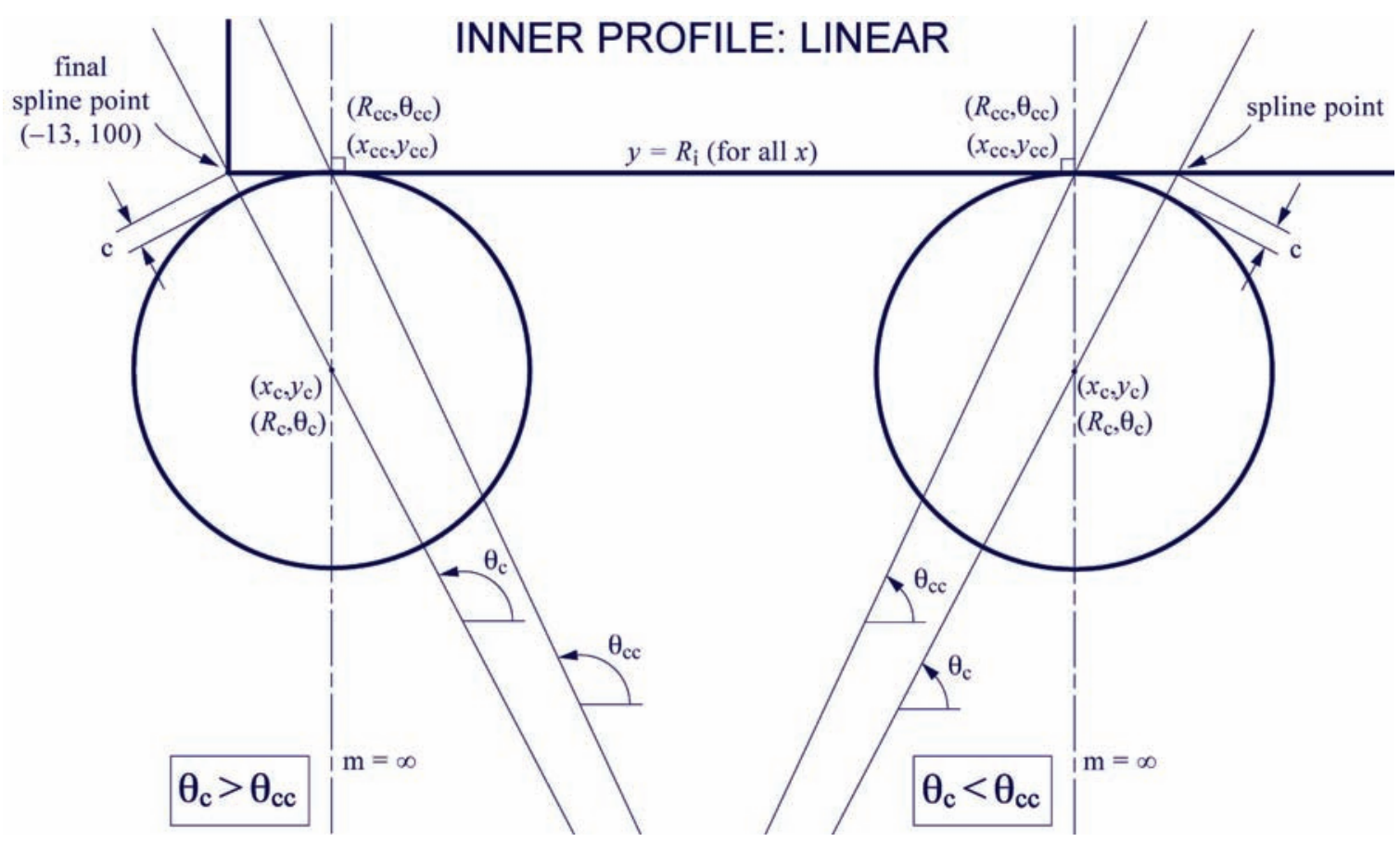

Figure 5-1. Details of the intersection of the inspection sphere and the horizontal line defining the inner profile for $\theta_{\mathrm{c}}>\theta_{\mathrm{cc}}$ (left) and $\theta_{\mathrm{c}}<\theta_{\mathrm{cc}}$ (right). 
As before, the solution for a general angle will be obtained by the simultaneous solution of the inner profile and the inspection sphere. The inner profile is given as,

$$
y_{\mathrm{cc}}=R_{\mathrm{i}} \quad \text { (for all } \mathrm{x}_{\mathrm{cc}} \text {, regardless of } \theta_{\mathrm{c}} \text { ). }
$$

The sphere is given in Eq. 3-2 as

$$
\left(x_{\mathrm{cc}}-x_{\mathrm{c}}\right)^{2}+\left(y_{\mathrm{cc}}-y_{\mathrm{c}}\right)^{2}=\phi^{2} / 4 .
$$

The derivative of the profile is

$$
d y / d x=\text { zero (horizontal line), }
$$

which implies from Figure 5-1 that

$$
x_{c c}=x_{c} \quad \text { and } \quad y_{c c}=y_{c}+\phi / 2 \text {. }
$$

Now,

$$
\theta_{\mathrm{c}}=\tan ^{-1}\left(y_{\mathrm{c}} / x_{\mathrm{c}}\right),
$$

so

$$
y_{\mathrm{c}}=x_{\mathrm{c}} \tan \theta_{\mathrm{c}} .
$$

Using Eq. 5-2, noting that $x_{c}=x_{c c}$ in Eq. 5-4, substituting $R_{i}$ for $y_{c c}$ from Eq. 5-1, and using the identity in Eq. 5-5, then

$$
\left(R_{\mathrm{i}}-x_{\mathrm{c}} \tan \theta_{\mathrm{c}}\right)^{2}=\phi^{2} / 4,
$$

so

$$
x_{\mathrm{c}} \tan \theta_{\mathrm{c}}=R_{\mathrm{i}}-\phi / 2 .
$$

Finally,

$$
x_{\mathrm{cc}}=x_{\mathrm{c}}=\frac{R_{\mathrm{i}}-\phi / 2}{\tan \theta_{\mathrm{c}}}
$$

and

$$
y_{\mathrm{cc}}=R_{\mathrm{i}}, x_{\mathrm{c}}=x_{\mathrm{cc}} \text {, and } y_{\mathrm{c}}=R_{\mathrm{i}}-\phi / 2 \text {. }
$$




\subsection{Outer linear profile}

This solution is only slightly more complicated than the one just completed in that the slope of the line is nonzero (see Figure 1-2). Figure 5-2 shows the intersection of the inspection sphere at three locations: $\theta_{\mathrm{c}}>\alpha_{1}, \theta_{\mathrm{c}}=\alpha_{1}$, and $\theta_{\mathrm{c}}<\alpha_{1}$. A s in the previous section, the solution is identical for all three cases.

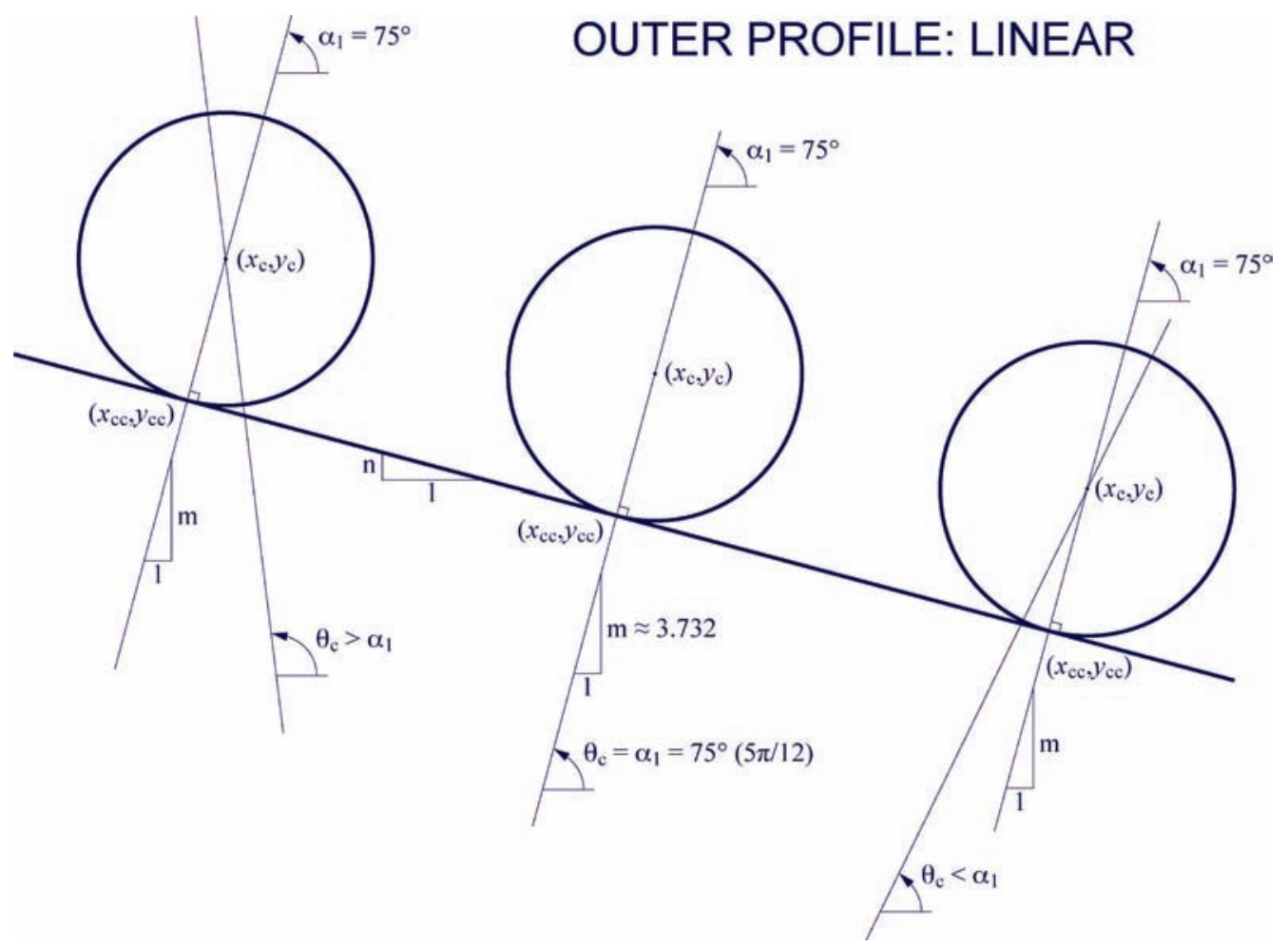

Figure 5-2. Details of the intersection of the inspection sphere and the linear portion of outer profile for $\theta_{\mathrm{c}}>\alpha_{1}$ (left), $\theta_{\mathrm{c}}=\alpha_{1}$ (middle), and $\theta_{\mathrm{c}}<\alpha_{1}$ (right).

The known quantities for this problem are

$\mathrm{R}_{0}, \mathrm{x}_{0}, \alpha_{1}, \phi, \mathrm{x}_{i}, \mathrm{x}_{\mathrm{d}}$, and $\theta_{\mathrm{c}}$ (the spline point angle, e.g., $\pi / 3$ ).

As a reminder from Chapter $1, \alpha_{1}$ is the angle normal to the slope of the straight-line section of the outer profile, i.e., $\pi / 2-\pi / 12=5 \pi / 12$, or 75 degrees. The values of $x_{i}$ and $x_{d}$ are the distances from the part center to the step and the additional distance to the edge of the outer profile, i.e., $13 \mathrm{~mm}$ and $2 \mathrm{~mm}$, respectively, as shown in Figure 1-2.

The unknowns are, as in the previous section,

$$
\mathrm{R}_{\mathrm{cc},}, \theta_{\mathrm{cc}}\left(\mathrm{x}_{\mathrm{cc},} \mathrm{y}_{\mathrm{cc}}\right) \text {, and } \mathrm{R}_{\mathrm{c}} \text {. }
$$

We begin by solving for the intersection of the inspection sphere and the outer profile, i.e., the nonhorizontal straight line defined by 


$$
y_{\mathrm{cc}}=\mathrm{n} x_{\mathrm{cc}}+\mathrm{b},
$$

The slope is denoted " $n$," since " $m$ " has already been used as the slope between the sphere center and the contact point in Eq. 3-3.

The slope of the outer profile is

$$
\Delta y / \Delta x=\mathrm{n}=\tan (-\pi / 12),
$$

where (from Eq. 1-20)

$$
\Delta y=\tan (\pi / 12) \cdot\left(x_{\alpha_{1}}+x_{0}\right) .
$$

The $y$-intercept is given by (Eq. 1-21),

$$
\mathrm{b}=y_{\alpha_{1}}+\Delta y
$$

and (from Eq. 1-17 and Eq. 1-16, respectively)

$$
y_{\alpha_{1}}=x_{\alpha_{1}} \tan \alpha_{1}
$$

and

$$
x_{\alpha_{1}}=\sqrt{R_{\mathrm{o}}^{2} /\left(1+\tan ^{2} \alpha_{1}\right)} .
$$

The inspection sphere is given above, in Eq. 5-2, as

$$
\left(x_{\mathrm{cc}}-x_{\mathrm{c}}\right)^{2}+\left(y_{\mathrm{cc}}-y_{\mathrm{c}}\right)^{2}=\phi^{2} / 4 .
$$

The line perpendicular to the profile connecting the contact point $\left(x_{c c}, y_{c c}\right)$ to the inspection sphere center $\left(x_{c}, y_{c}\right)$ is

$$
\mathrm{m}=\Delta y / \Delta x=\left(y_{\mathrm{cc}}-y_{\mathrm{c}}\right) /\left(x_{\mathrm{cc}}-x_{\mathrm{c}}\right)=-1 / \mathrm{n}=1 / \tan (\pi / 12),
$$

so

$$
\left(y_{\mathrm{cc}}-y_{\mathrm{c}}\right)^{2}=\frac{\left(x_{\mathrm{cc}}-x_{\mathrm{c}}\right)^{2}}{\tan ^{2}(\pi / 12)}=\left(x_{\mathrm{cc}}-x_{\mathrm{c}}\right)^{2} \mathrm{~m}^{2} .
$$

Or using Eq. 5-17,

$$
\frac{\left(x_{\mathrm{cc}}-x_{\mathrm{c}}\right)^{2}}{\tan ^{2}(\pi / 12)}+\left(x_{\mathrm{cc}}-x_{\mathrm{c}}\right)^{2}=\phi^{2} / 4
$$




$$
\left(x_{\mathrm{cc}}-x_{\mathrm{c}}\right)^{2}\left[1+\frac{1}{\tan ^{2}(\pi / 12)}\right]=\phi^{2} / 4
$$

so

$$
x_{\mathrm{cc}}-x_{\mathrm{c}}=\frac{ \pm \phi / 2}{\sqrt{\left[1+\tan ^{2}(\pi / 12)\right]}}=\frac{ \pm \phi / 2}{\sqrt{1+\mathrm{m}^{2}}},
$$

or, taking only the negative root in a modified form of $E q .5-22$ (since $x_{c}$ is al ways greater than $x_{c c}$ on the outer profile, as shown in Figure 5-2),

$$
x_{\mathrm{c}}=x_{\mathrm{cc}}+\frac{\phi / 2}{\sqrt{\left[1+\tan ^{2}(\pi / 12)\right]}}=x_{\mathrm{cc}}+\frac{\phi / 2}{\sqrt{1+\mathrm{m}^{2}}} .
$$

Also, from Figure 5-2 (and by definition)

$$
\tan \theta_{\mathrm{c}}=y_{\mathrm{c}} / x_{\mathrm{c}}
$$

so

$$
y_{\mathrm{c}}=x_{\mathrm{c}} \tan \theta_{\mathrm{c}} \text { and } x_{\mathrm{c}}=y_{\mathrm{c}} / \tan \theta_{\mathrm{c}} .
$$

From Eq. 5-18,

$$
y_{\mathrm{cc}}=y_{\mathrm{c}}+\mathrm{m}\left(x_{\mathrm{cc}}-x_{\mathrm{c}}\right) \text {, }
$$

and from Eq. 5-11, substituting $n$ for $-1 / m$,

$$
y_{\mathrm{cc}}=\mathrm{n} x_{\mathrm{cc}}+\mathrm{b}=\mathrm{b}-\frac{x_{\mathrm{cc}}}{\mathrm{m}} \text {, }
$$

SO

$$
y_{\mathrm{c}}+\mathrm{m}\left(x_{\mathrm{cc}}-x_{\mathrm{c}}\right)=\mathrm{b}-\frac{x_{\mathrm{cc}}}{\mathrm{m}},
$$

or

$$
x_{\mathrm{c}} \tan \theta_{\mathrm{c}}+\mathrm{m}\left(x_{\mathrm{cc}}-x_{\mathrm{c}}\right)=\mathrm{b}-\frac{x_{\mathrm{cc}}}{\mathrm{m}},
$$

or

$$
x_{\mathrm{c}} \tan \theta_{\mathrm{c}}-\mathrm{m} x_{\mathrm{c}}=\mathrm{b}-\frac{x_{\mathrm{cc}}}{\mathrm{m}}-\mathrm{m} x_{\mathrm{c}},
$$


or

$$
x_{\mathrm{c}}\left(\tan \theta_{\mathrm{c}}-\mathrm{m}\right)=\mathrm{b}-x_{\mathrm{cc}}(\mathrm{m}+1 / \mathrm{m})
$$

and therefore

$$
x_{\mathrm{c}}=\frac{\mathrm{b}-x_{\mathrm{cc}}(\mathrm{m}+1 / \mathrm{m})}{\tan \theta_{\mathrm{c}}-\mathrm{m}} \text {. }
$$

But using the definition of $x_{c}$ in Eq. 5-23,

$$
\frac{\mathrm{b}-x_{\mathrm{cc}}(\mathrm{m}+1 / \mathrm{m})}{\tan \theta_{\mathrm{c}}-\mathrm{m}}=x_{\mathrm{cc}}+\frac{\phi / 2}{\sqrt{1+\mathrm{m}^{2}}},
$$

or

$$
\frac{-x_{\mathrm{cc}}(\mathrm{m}+1 / \mathrm{m})}{\tan \theta_{\mathrm{c}}-\mathrm{m}}-x_{\mathrm{cc}}=\frac{\phi / 2}{\sqrt{1+\mathrm{m}^{2}}}-\frac{\mathrm{b}}{\tan \theta_{\mathrm{c}}-\mathrm{m}}
$$

or

$$
x_{\mathrm{cc}}\left[\frac{-(\mathrm{m}+1 / \mathrm{m})}{\tan \theta_{\mathrm{c}}-\mathrm{m}}-1\right]=\frac{\phi / 2}{\sqrt{1+\mathrm{m}^{2}}}-\frac{\mathrm{b}}{\tan \theta_{\mathrm{c}}-\mathrm{m}}
$$

and finally,

$$
x_{\mathrm{cc}}=\frac{1}{\left[\frac{-(\mathrm{m}+1 / \mathrm{m})}{\tan \theta_{\mathrm{c}}-\mathrm{m}}-1\right]}\left[\frac{\phi / 2}{\sqrt{1+\mathrm{m}^{2}}}-\frac{\mathrm{b}}{\tan \theta_{\mathrm{c}}-\mathrm{m}}\right] \text {. }
$$

N ow that $x_{c c}$ has been solved, $y_{c c}$ can be solved from Eq. 5-27. Similarly, $x_{c}$ can be solved using Eqs. 5-32 and/or 5-23. Finally, $y_{c}$ is solved from Eq. 5-25. It is useful to have $y_{c}$ in terms of $x_{c c}$ and $x_{c}$, so using Eq. 5-28,

$$
y_{\mathrm{c}}=\mathrm{b}-\frac{x_{\mathrm{cc}}}{\mathrm{m}}-\mathrm{m}\left(x_{\mathrm{cc}}-x_{\mathrm{c}}\right)
$$

A this point, all the unknown variables have been solved on the straight-line section of the outer profile. 


\subsection{Solution for minimum component values}

From Figure 1-1, the minimum $x$-value for the inner profile (not considering the step) occurs on the straight-line (horizontal) surface at $\theta_{2}$.

Here, $y_{c c}=R_{i}(100 \mathrm{~mm})$ and $x_{c c}=-x_{i}(-13 \mathrm{~mm})$. By inspection of Figure 1-1,

$$
\theta_{2}(\text { inner })=\pi / 2+\tan ^{-1}\left(-x_{\mathrm{i}} / R_{\mathrm{i}}\right),
$$

so

$\theta_{2}$ (inner) is approximately 97.406912 degrees; $x_{c c}$ is $-12.74203 \mathrm{~mm}>x_{i}(-13 \mathrm{~mm})$.

Therefore, this is a valid point and may be inspected as illustrated in Figure 5-1.

For the outer profile, the maximum $y$-value $\left(\mathrm{y}_{\text {sharp }}\right)$ on the component was determined from Eq. 1-24 to be approximately $115.402729 \mathrm{~mm}$. This agrees with the drawing in A ppendix A. The angle associated with this value, as shown in Figure 1-2, would be

$$
\theta_{2}(\text { outer })=\frac{\pi}{2}+\tan ^{-1}\left(\frac{x_{\mathrm{i}}+x_{\mathrm{d}}}{y_{\text {sharp }}}\right) .
$$

This leads to the approximate values of $\theta_{2}$ of 97.40576 degrees and $x_{c c}$ of $-15.7902566 \mathrm{~mm}$. This contact point would be off the part $\left(x_{c c}<-\left(x_{i}+x_{d}\right)=-15\right)$, as illustrated in Figure 5-2 $\left(\theta_{c}>\alpha_{1}\right)$. The question then may be posed to find the maximum contact angle on the part. By substituting $x_{c c}=-15$ and solving for $x_{c}$ and $y_{c}$, that value is approximately 97.039153 degrees. It is important to have these numbers in order to determine the limits of the cosine correction value.

\subsection{Cosine correction}

The cosine correction was defined at the beginning of Chapter 2 as the difference between the radius to the sphere edge along the spline angle at the contact point and the radius of the spline point at any arbitrary angle $\theta: c=\left(R_{c c}\right)_{\theta}-R_{\theta}$. Now that the radii for the inner and outer profiles have been completely defined, for their circular and straight-line segments, the cosine correction may be determined for all angles. Figure 5-3 shows these values as a function of spline angle. B oth curves show a linear portion that changes to parabolic at the transition to the straight-line portion of each curve. B oth curves pass through zero (when $\theta_{\mathrm{c}}=$ $\left.\theta_{c c}\right)$ for angles of $\pi / 2$ and $\alpha_{1}$ for the inner and outer profiles, respectively. 


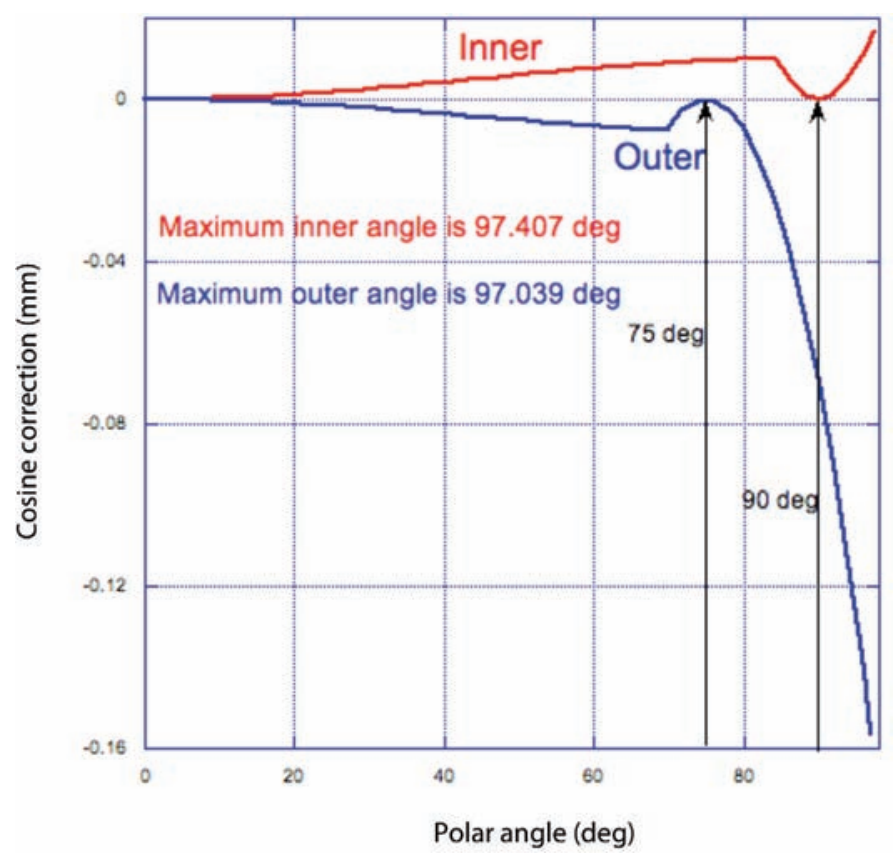

Figure 5-3. Cosine correction, $c=\left(R_{\mathrm{cc}}\right)_{\theta}-R_{\theta}$, for the inner (red) and outer (blue) profiles. 


\section{Chapter 6. Component volumes}

Chapters 1 to 5 presented analytic methods to define the various surfaces of the CM M Test A rtifact and its interaction with a theoretical inspection sphere. It is of some interest to compare the total volume of the part, at different levels of complexity, e.g., with and without the step, calculated by three different methods: the analytic description, a numerical approximation (using ten million slices), and a ProE model. This section is dedicated to that assessment.

\subsection{Outer profile volume}

Figure 6-1 shows a representation of the volume enclosed by the outer contour, i.e., the "outer volume." It consists of two coaxial cones and the segment of a sphere. The base of the large outer cone, shown in Figure 6-1, has a radius equal to ysharp, defined in Eq. 1-24,

$$
y_{\text {sharp }}=y_{\alpha_{1}}+\Delta y^{\prime} \approx 115.402729 \mathrm{~mm} \text {, }
$$

where $\Delta \mathrm{y}^{\prime}$ is defined in Eq. 1-23 as

$$
\Delta y^{\prime}=-\tan (-\pi / 12) \cdot\left(x_{\alpha_{1}}+10+13+2\right) \approx 13.980517 \mathrm{~mm} .
$$

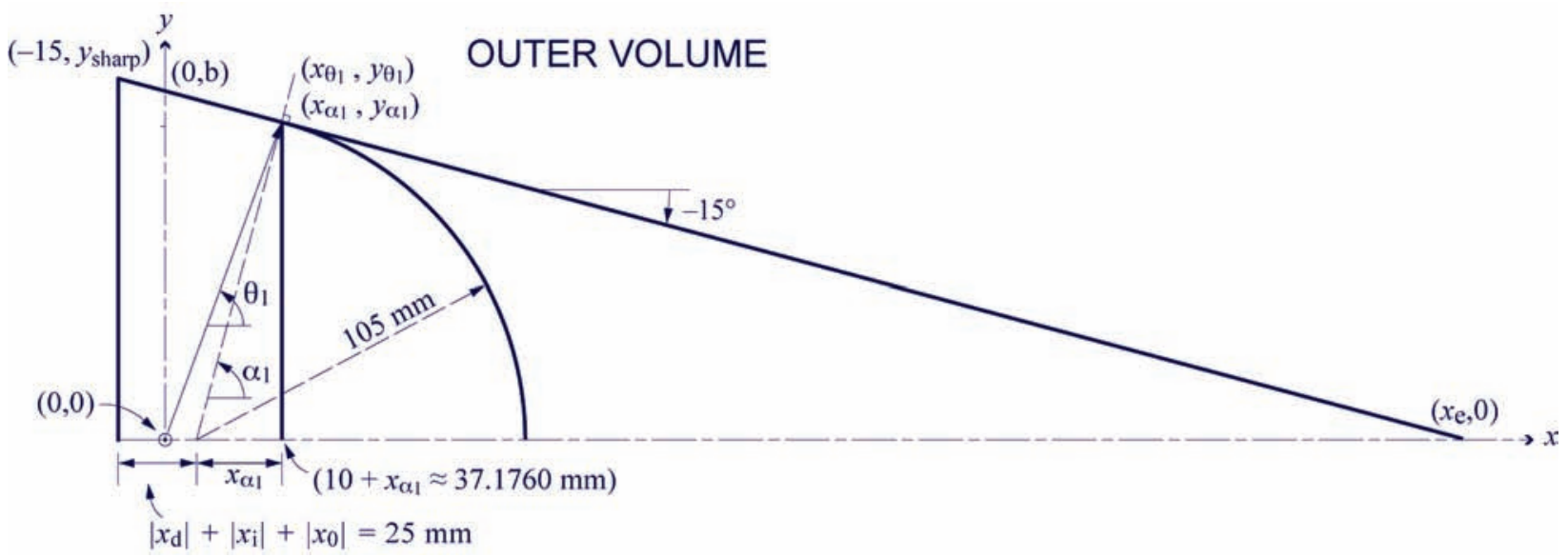

Figure 6-1. Outer cone, inner cone, and spherical segment used to define the analytic volume of the outer profile.

The values of 10,13 , and 2 are the values of $x_{0}, x_{i}$, and $x_{d}$, respectively. The intersection of the straight line section and the circular section of the outer profile is at $\left(x_{\alpha_{1}}, y_{\alpha_{1}}\right)$. These coordinates are given in Eqs. 1-16 and 1-17, respectively:

$$
\begin{aligned}
& x_{\alpha_{1}}=\sqrt{R_{\mathrm{o}}^{2} /\left(1+\tan ^{2} \alpha_{1}\right)}, \\
& y_{\alpha_{1}}=x_{\alpha_{1}} \tan \alpha_{1} .
\end{aligned}
$$


Note that intersection point in the $\theta$ and $\alpha$ references is the same and that

$$
x_{\theta_{1}}=x_{\alpha_{1}}+x_{0}
$$

and

$$
y_{\theta_{1}}=y_{\alpha_{1}} .
$$

In order to find the vertex of the cone, note that in Figure $6-1, \Delta y / \Delta x=\tan (-\pi / 12), \Delta x$ and $\Delta y$ may be found from $\left(x_{\alpha_{1}}, y_{\alpha_{1}}\right)$, where

$$
\Delta y=-y_{\alpha_{1}}
$$

and

$$
\Delta x=\frac{-y_{\alpha_{1}}}{\tan (-\pi / 12)} \approx 378.5128 \mathrm{~mm} .
$$

The vertex of both cones is located at a point $\left(x_{e}, 0\right)$, where

$$
x_{\mathrm{e}}=x_{0}+x_{\alpha_{1}}+\Delta x \approx 415.6888 \mathrm{~mm} \text {. }
$$

Figure 6-2 illustrates the heights of the cones. The small cone height is

$$
h_{\mathrm{c}_{2}}=x_{\mathrm{e}}-x_{\alpha_{1}}-x_{0},
$$

and the large cone height is

$$
h_{\mathrm{c}_{1}}=h_{\mathrm{c}_{2}}+x_{\alpha_{1}}+x_{0}+\left|x_{\mathrm{i}}\right|+\left|x_{\mathrm{c}}\right|=x_{\mathrm{e}}+\left|x_{\mathrm{i}}\right|+\left|x_{\mathrm{c}}\right| .
$$

The volume of the outer profile of the analytic part is

$$
V_{\text {total }}=V_{\text {cone1 }}-V_{\text {cone2 }}+V_{\text {seg }}
$$

where the value of the spherical segment is illustrated by the shaded area in Figure 6-2.

If $h_{1}$ in Figure $6-2$ is given as

$$
h_{1}=R_{0}-x_{\alpha_{1}},
$$

then the volume of the spherical segment is 


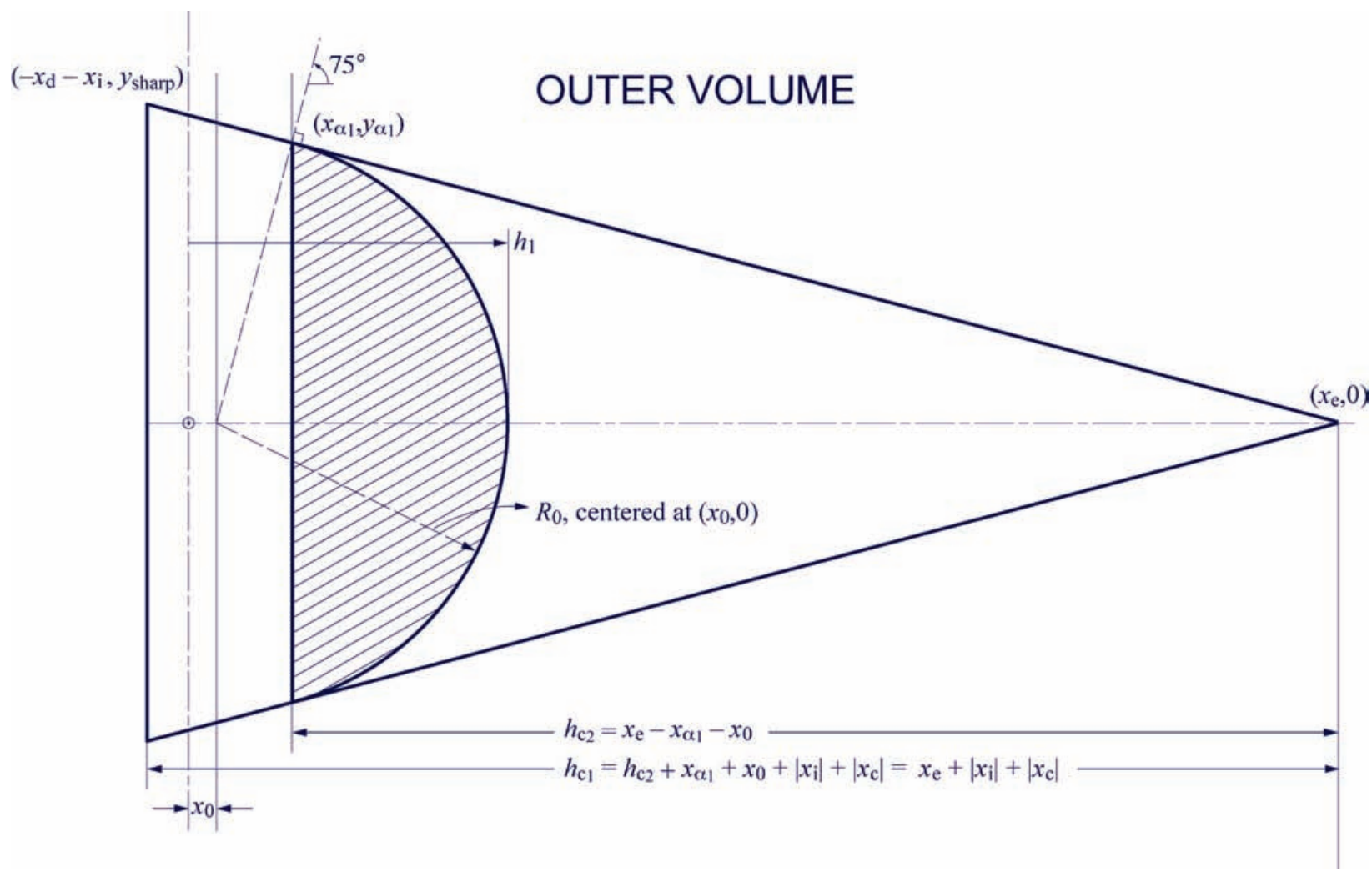

Figure 6-2. Details of the dimensions of the conic sections in Figure 6-1.

$$
V_{\text {seg }}=\frac{\pi}{3} h_{1}^{2}\left(3 R_{0}-h_{1}\right) \approx 1,504,272.301 \mathrm{~mm}^{3} .
$$

The base of the large outer cone is $y_{\text {sharp }}$ (Eq. 6-1), and the height is $h_{c_{1}}$ (Eq. 6-11). So its volume is given by

$$
V_{\text {cone1 }}=\frac{\pi}{3} y_{\text {sharp }}^{2} h_{c_{1}} \approx 6,006,540.367 \mathrm{~mm}^{3} \text {. }
$$

The diameter of the smaller cone is $y_{\alpha_{1}}$ (Eq. 6-4), and the height is $h_{c_{2}}$ (Eq. 6-10). So its volume is given by

$$
V_{\text {cone2 }}=\frac{\pi}{3} y_{\alpha_{1}}^{2} h_{c_{2}} \approx 4,077,325.635 \mathrm{~mm}^{3} \text {. }
$$

Finally, the total outer volume can be calculated (Eq. 6-12), 3,433,487.033 $\mathrm{mm}^{3}$. 


\subsection{Inner profile volume}

The volume of the inner profile is considerably easier to calculate. The volume consists of a quarter-sphere and a cylinder, as shown in Figure 6-3. The radius of both is $R_{i}$, and the thickness of the cylinder is the distance from the part center to the edge of the part. The volumes are

$$
V_{\text {hemi, inner }}=\frac{2 \pi}{3} R_{\mathrm{i}}^{3}
$$

and

$$
V_{\text {cyl, inner }}=\pi R_{\mathrm{i}}^{2}\left(x_{0}+\left|x_{\mathrm{i}}\right|+\left|x_{\mathrm{c}}\right|\right) .
$$

The total inner volume is the sum of these terms, $\sim 2,879,793.267 \mathrm{~mm}^{3}$. The volume of the component (without the step and the rounds) is therefore the difference of the outer profile and the inner profile, $\sim 553,693.766 \mathrm{~mm}^{3}$.

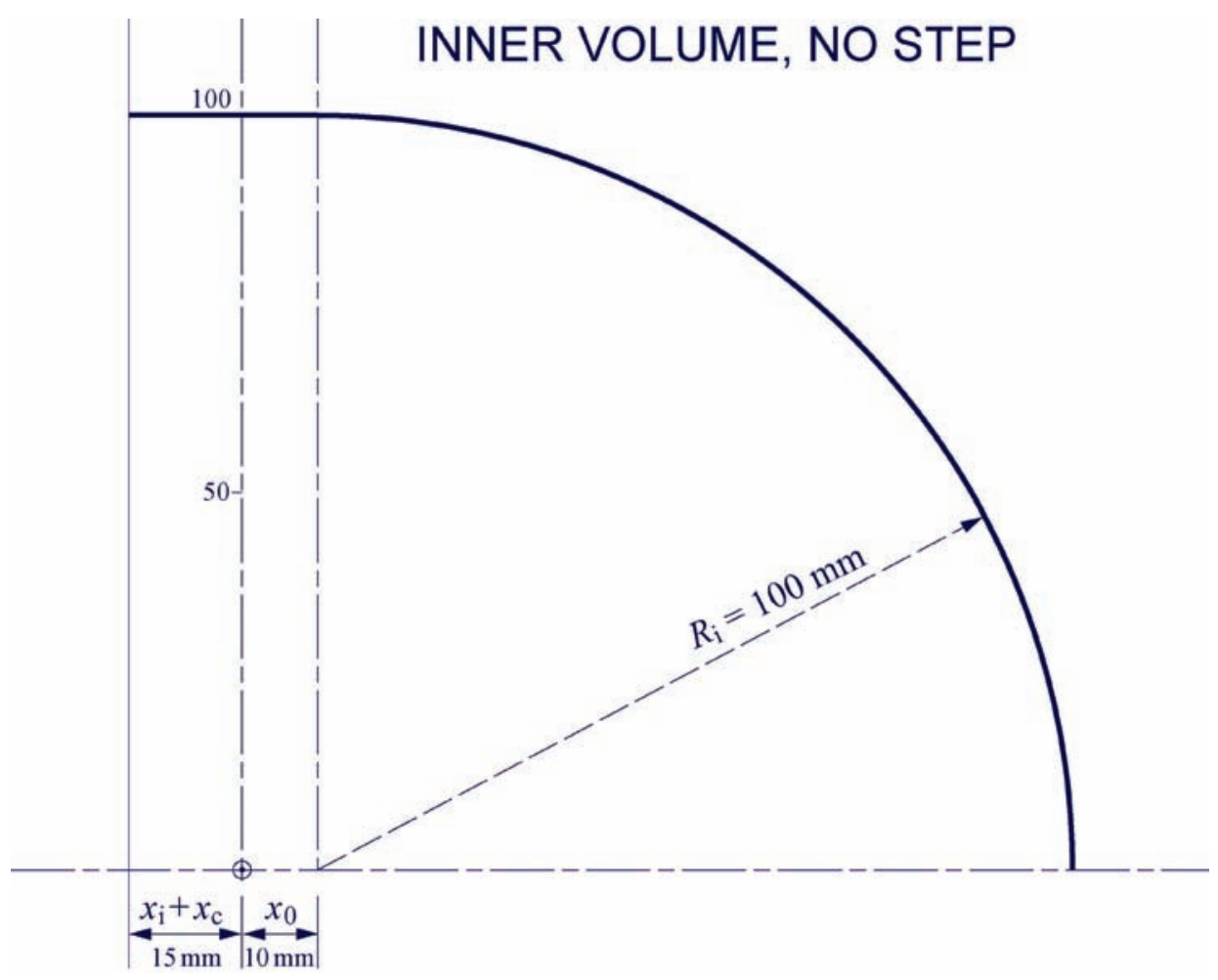

Figure 6-3. Geometric description of the inner profile, ignoring the step. 


\subsection{Step volume}

A representation of the step is shown in Figure 6-4, taken from Figure 1-3. The volume of any arbitrary plane area, $\mathbf{A}$, about an axis in its plane is,

$$
\mathrm{V}=2 \pi \mathbf{R A}
$$

where $\mathbf{R}$ is the distance of the center of gravity from the axis. From Figure 6-4, the area of the rectangle is $20 \mathrm{~mm}^{2}$, and the center of gravity, by inspection, is $105 \mathrm{~mm}$. The step volume is therefore

$$
V_{\text {step }}=2 \pi(105)(20) \approx 13,194.68915 \mathrm{~mm}^{3} .
$$

This volume will be subtracted from the component calculated above.

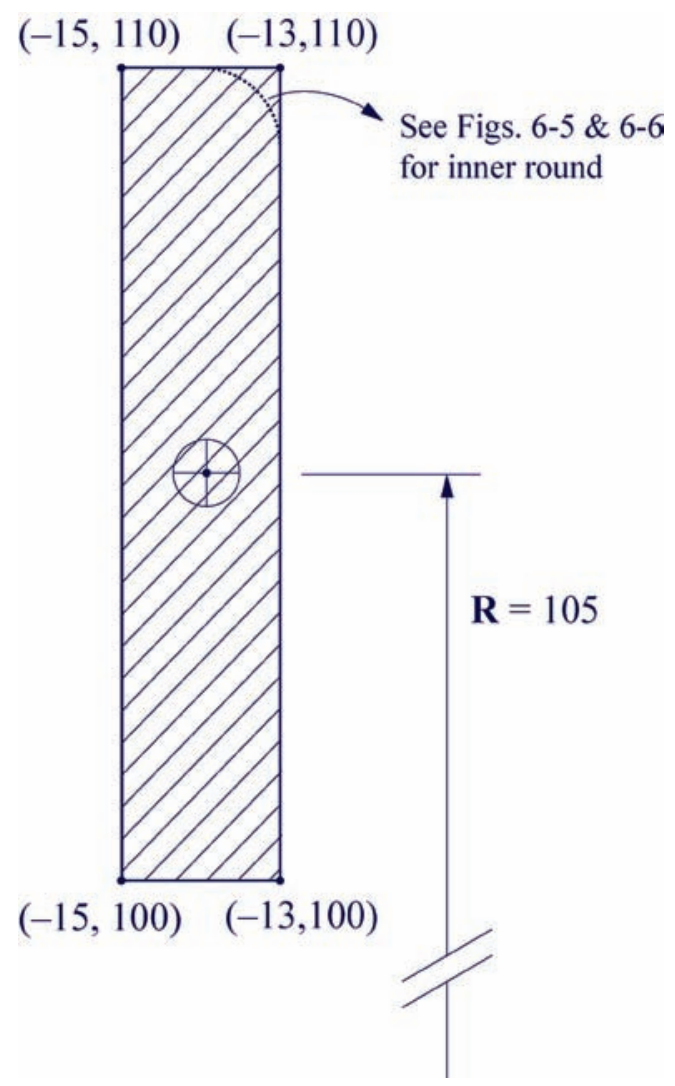

Figure 6-4. Geometric description of the step. 


\subsection{Inner round volume}

Detail $C$ of the part definition in A ppendix A calls out two "rounds" that specify radii on two different corners of the component. The inner radius is $0.25 \mathrm{~mm}(\max )$ and is at the corner of the outer edge of the step. It is called the "inner" round here because it is closer to the axis of rotation than the other round that is discussed in the next section. Figure 6-5 illustrates the circular section under consideration. Datum $A$ is shown that matches the datum in A ppendix A. In order to simplify the volume solution, the origin is considered to be the theoretical corner. It is clear that the shaded region will add volume to the component $(R, 0)$. The area above the shaded area in Figure 6-5 is found by

$$
A=\int\left(a x^{2}+b\right)^{1 / 2} \mathrm{~d} x=\frac{x}{2}\left(a x^{2}+b\right)^{1 / 2}+\left.\frac{b}{2(-a)^{1 / 2}} \sin ^{-1}\left[x\left(\frac{-a}{b}\right)^{1 / 2}\right]\right|_{0} ^{R}
$$

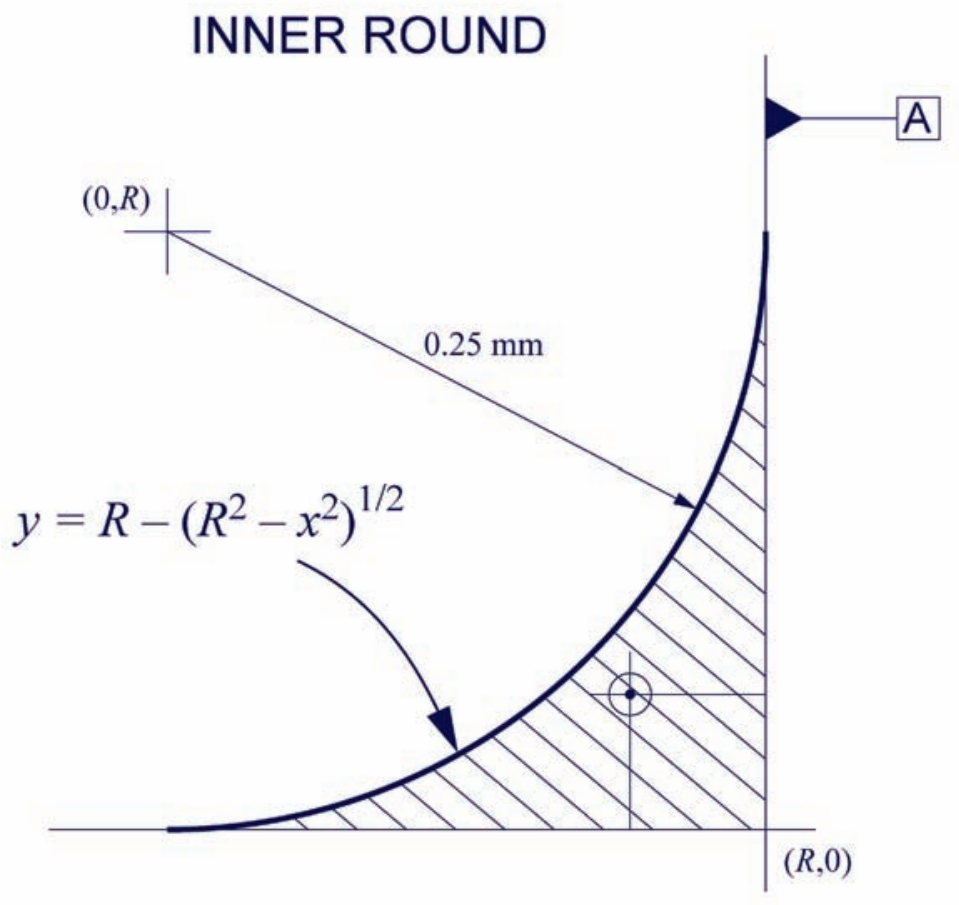

Figure 6-5. Simplified representation of the inner round.

Here, $a=-1$ and $b=R^{2}$, so

$$
A=\frac{x}{2}\left(R^{2}-x^{2}\right)^{1 / 2}+\left.\frac{R^{2}}{2} \sin ^{-1}\left(\frac{x}{R}\right)\right|_{0} ^{R}
$$

The area of interest (the shaded region) is 


$$
A_{\mathrm{i}}=R^{2}-\frac{\pi R^{2}}{4}=R^{2}\left(1-\frac{\pi}{4}\right) \approx 0.01341265 \mathrm{~mm}^{2} .
$$

A ssuming, for the time being, that the lower boundary of the shaded area of Figure $6-5$ is $y=0$, the center of gravity of this section is

$$
x_{\mathrm{cg}}=\frac{\int_{0}^{R} x y \mathrm{~d} x}{\int_{0}^{R} y \mathrm{~d} x}=\frac{\int_{0}^{R} x y \mathrm{~d} x}{A_{\mathrm{i}}}
$$

where $A_{i}$ is defined in Eq. 6-23. Since in Figure 6-5

$$
y=R-\left(R^{2}-x^{2}\right)^{1 / 2} \text {, choosing the negative root, }
$$

then

$$
\begin{aligned}
\int_{0}^{R} x y \mathrm{~d} x & =\int_{0}^{R} x\left[R-\left(R^{2}-x^{2}\right)^{1 / 2}\right] \mathrm{d} x=\int_{0}^{R} x R \mathrm{~d} x-\int_{0}^{R} x\left(R^{2}-x^{2}\right)^{1 / 2} \mathrm{~d} x \\
& =\left.\frac{R x^{2}}{2}\right|_{0} ^{R}+\left.\frac{\left(R^{2}-x^{2}\right)^{3 / 2}}{3}\right|_{0} ^{R}=\frac{R^{3}}{6}
\end{aligned}
$$

so

$$
x_{\mathrm{cg}}=\frac{R}{6(1-\pi / 4)}
$$

and

$$
y_{\mathrm{cg}}=R\left[1-\frac{1}{6(1-\pi / 4)}\right] \approx 0.055841985 \mathrm{~mm}(\text { for } \mathrm{R}=0.25 \mathrm{~mm})
$$

Given that the base in Figure 6-5 (from A ppendix A), $y_{c i}$, is $110 \mathrm{~mm}$, then the step may be visualized more completely in Figure 6-6. Then from Eq. 6-19,

$$
V_{\text {inner, round }}=2 \pi A_{\mathrm{i}}\left(y_{\mathrm{ci}}-y_{\mathrm{cg}}\right) \approx 9.265427831 \mathrm{~mm}^{3} \text {. }
$$




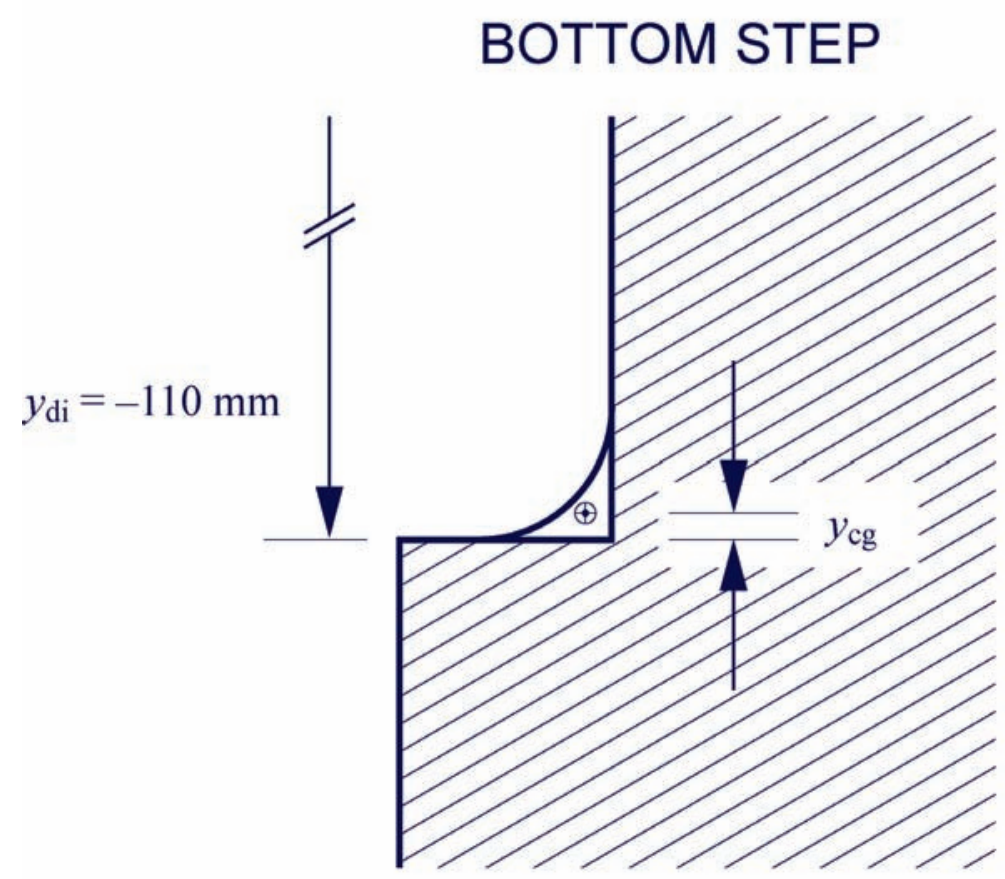

Figure 6-6. Illustration of step in the component geometry.

\subsection{Outer round volume}

Detail $C$ of the component drawing in A ppendix $A$ also defines a round with a radius of 0.8 $\mathrm{mm}$ at the most extreme $y$-value ( $y_{\text {sharp }}$ ) of the part. This is a more complex shape than the inner round and is shown in Figure 6-7 as the combination of four smaller shapes:

$$
V_{\text {outer round }}=V_{1}+V_{3}+V_{4}-V_{2} \text {. }
$$

The volumes are calculated in a two-dimensional coordinate system with the origin $(0,0)$ located at circled point A. Circled point C corresponds to the "sharp" point shown in Figure 1-3. The local coordinates of the five points necessary to calculate the areas are given on Figure 6-7.

A $n$ approach utilizing Eq. 6-19 is used to solve for the individual volumes. The coordinate system to calculate the moment arm ( $\mathbf{R}$ in Eq. 6-19) must be linked to the component center.

\section{Volume 1 (rectangle)}

$$
\begin{aligned}
& A_{1}=R^{2} \cos (\pi / 12), \\
& y_{\mathrm{cg} 1}=y_{\mathrm{k}}+\frac{R \cos (\pi / 12)}{2},
\end{aligned}
$$


where, as shown in Figure 6-7,

$$
y_{\mathrm{k}}=y_{\text {sharp }}-y_{\odot} .
$$

Therefore, from Eq. 6-19,

$$
V_{1}=2 \pi\left[y_{\mathrm{k}}+\frac{R \cos (\pi / 12)}{2}\right]\left[R^{2} \cos (\pi / 12)\right] \approx 445.7005193 \mathrm{~mm}^{3} .
$$

\section{Volume 2 (spherical sector)}

If $\alpha$ is the magnitude of the sector angle,

$$
A_{2}=\pi R^{2} \frac{\alpha}{2 \pi}=\frac{7 \pi R^{2}}{24}
$$

and

$$
y_{\mathrm{cg} 2}=y_{\mathrm{k}}+\frac{R[1-\cos (2 \alpha)]}{3 \alpha}=y_{\mathrm{k}}+\frac{R[1-\cos (7 \pi / 12)]}{7 \pi / 8} .
$$

So from Eq. 6-19,

$$
V_{2}=2 \pi\left\{y_{\mathrm{k}}+\frac{R[1-\cos (7 \pi / 12)]}{7 \pi / 8}\left[\frac{7 \pi R^{2}}{24}\right]\right\} \approx 442.72725255 \mathrm{~mm}^{3} .
$$

\section{Volume 3 ("top" triangle)}

From the definitions of $D_{x}, B_{y}$, and $C_{y}$ in Figure 6-7,

$$
A_{3}=\frac{1}{2}\left(\mathrm{C}_{y}-\mathrm{B}_{y}\right) \mathrm{D}_{x}=\frac{R^{2}}{2} \tan (\pi / 12)[1+\sin (\pi / 12)]^{2},
$$

and

$$
y_{\mathrm{cg} 3}=y_{\mathrm{k}}+\frac{\mathrm{C}_{y}-\mathrm{B}_{y}}{3}=y_{\mathrm{k}}+R\left\{\cos (\pi / 12)+\frac{\tan (\pi / 12)[1+\sin (\pi / 12)]}{3}\right\} \text {. }
$$




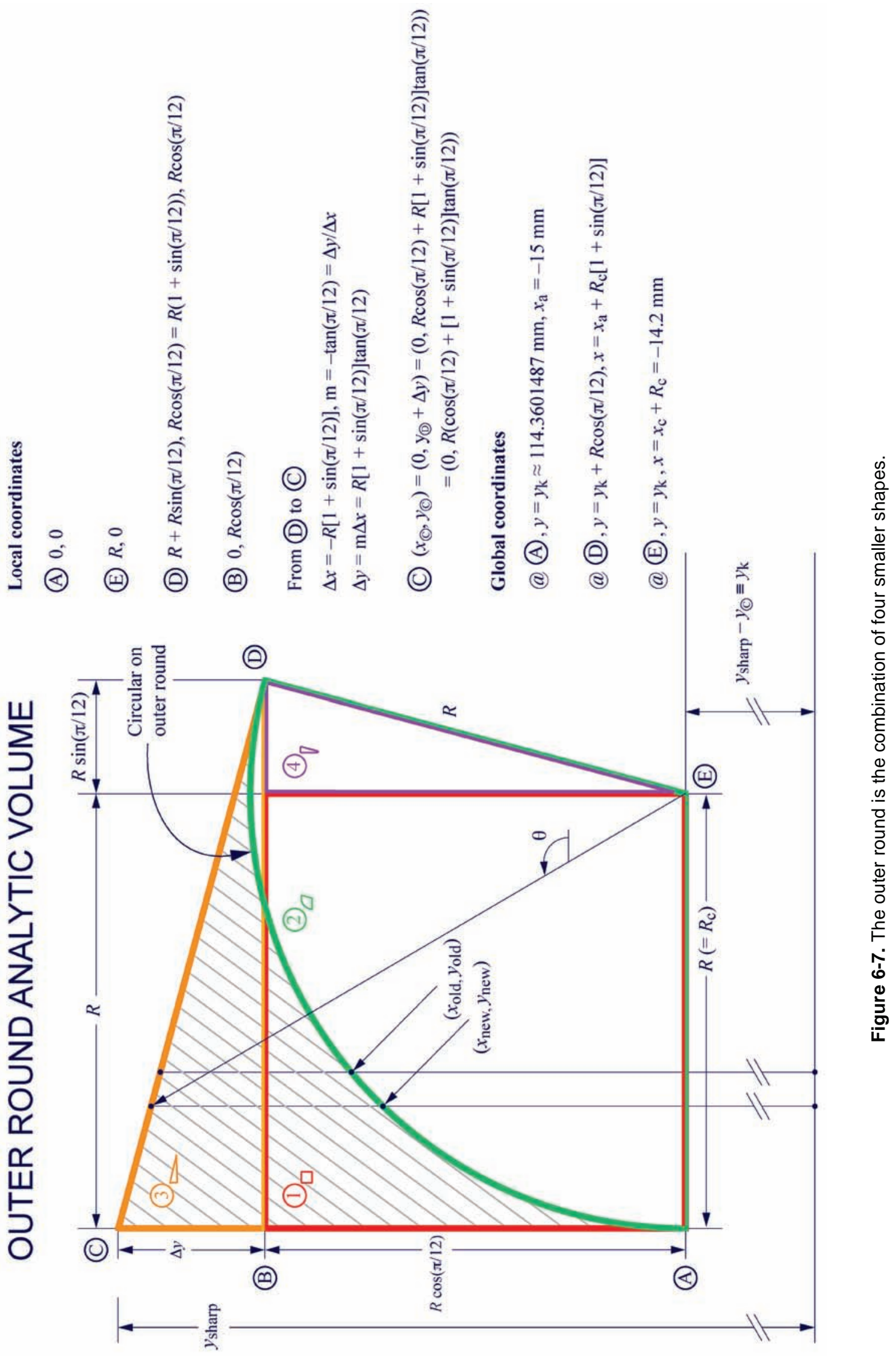


So from Eq. 6-19,

$$
\begin{aligned}
V_{3}=2 \pi\left(y_{\mathrm{k}}\right. & \left.+R\left\{\cos (\pi / 12)+\frac{\tan (\pi / 12)[1+\sin (\pi / 12)]}{3}\right\}\right) \\
& \times\left(\frac{R^{2}}{2} \tan (\pi / 12)[1+\sin (\pi / 12)]^{2}\right) \approx 98.36655664 \mathrm{~mm}^{3} .
\end{aligned}
$$

\section{Volume 4 ("right" triangle)}

From the definitions of $D_{y}, D_{x}$, and $E_{x}$ in Figure 6-7,

$$
y_{\mathrm{cg} 4}=y_{\mathrm{k}}+\frac{2}{3}\left(\mathrm{D}_{y}=\mathrm{B}_{y}\right)=y_{\mathrm{k}}+\frac{2 R}{3} \cos (\pi / 12),
$$

and

$$
A_{4}=\frac{1}{2}\left(\mathrm{D}_{y}=\mathrm{B}_{y}\right)\left(\mathrm{D}_{x}-\mathrm{E}_{x}\right)=\frac{R^{2}}{2} \sin (\pi / 12) \cos (\pi / 12) .
$$

A gain, from Eq. 6-19,

$$
V_{4}=2 \pi\left[y_{\mathrm{k}}+\frac{2 R}{3} \cos (\pi / 12)\right]\left[\frac{R^{2}}{2} \sin (\pi / 12) \cos (\pi / 12)\right] \approx 57.74262835 \mathrm{~mm}^{3} .
$$

Finally, then, from Eq. 6-31, the net analytic volume of the outer round is approximately $179.08245 \mathrm{~mm}^{3}$.

\subsection{Numerical approximation}

To help confirm the analytic solution above, a numerical approach was developed to calculate the volumes of individual slices (the outer profile minus the inner profile) and keep a tally of the sum. The treatment utilizes a trapezoidal approximation to the curve and equations developed in Chapter 1 for the profiles (see, for example Figure 1-4). The step and inner round are determined numerically from the prescription shown in Figure 6-8. Using the definitions in that figure, the circle is defined as,

$$
\left(x-x_{\mathrm{sc}}\right)^{2}+\left(y-y_{\mathrm{sc}}\right)^{2}=R_{\mathrm{ci}}^{2}
$$

where $\left(x_{s c}, y_{s c}\right)$ is the center of the inner radius circle, $x$ is known (incrementing al ong the $x$ axis), and the new $y$-value is solved by 


$$
y_{\text {snew }}=y_{\mathrm{sc}}+\sqrt{R_{\mathrm{ci}}^{2}-\left(x_{\mathrm{snew}}-x_{\mathrm{sc}}\right)^{2}}
$$

Similarly, Figure 6-7 illustrates the incremental numerical steps for cal culating the volume slice increments on the outer round, where

$$
y_{\mathrm{n}}=y_{\mathrm{k}}+\sqrt{R_{\mathrm{c}}^{2}-\left[x_{\mathrm{n}}-\left(x_{\mathrm{c}}+r_{\mathrm{c}}\right)\right]^{2}}
$$

\section{INNER ROUND NUMERICAL VOLUME}
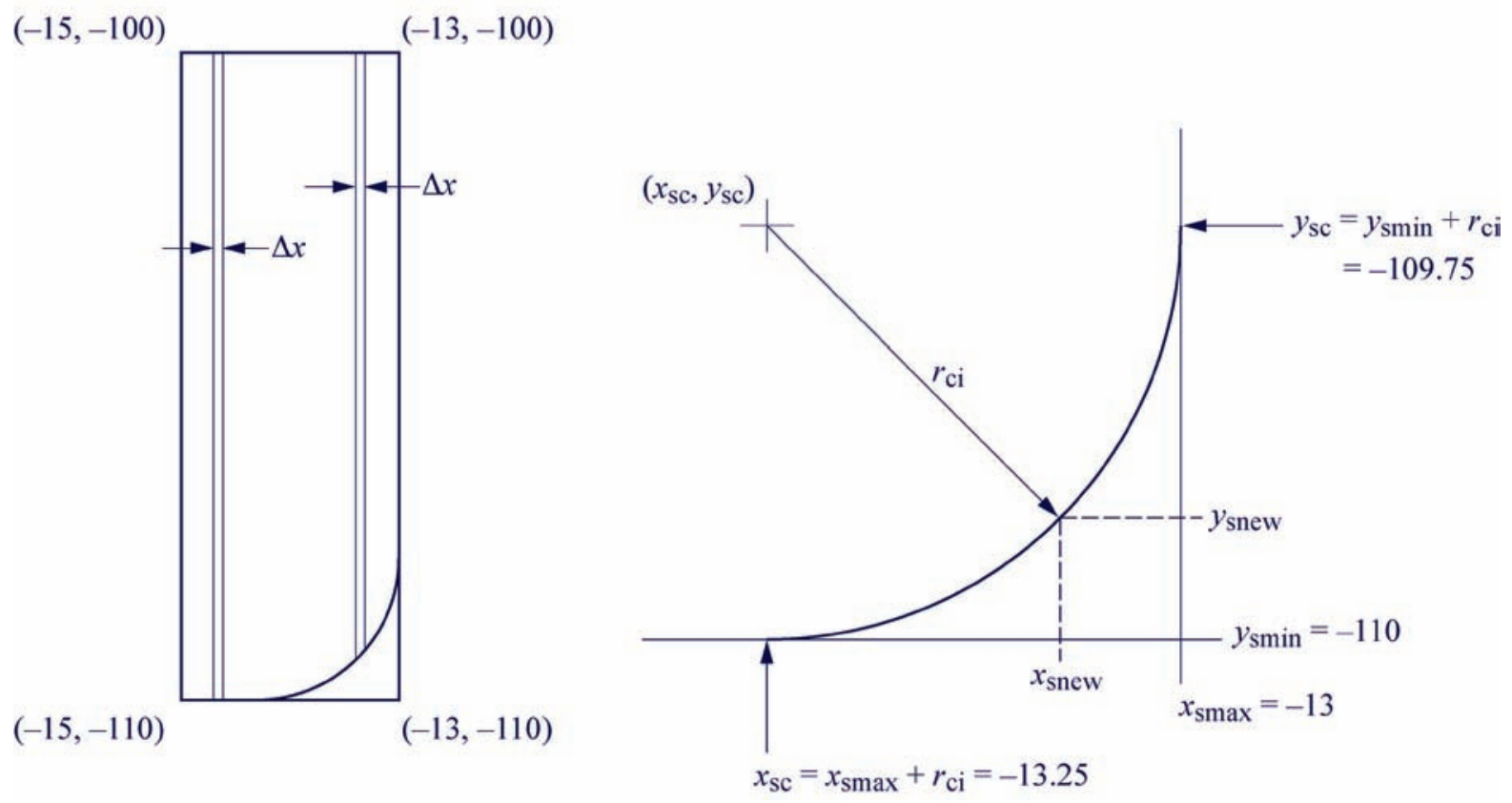

Figure 6-8. The step-wise numerical approximation of the step and inner round.

Table 6-1 shows the convergence of the outer and inner part volumes (without the step and rounds) and the outer round and the inner round volumes as the number of slices increases to ten million (double-precision accuracy).

Table 6-1. Convergence of numerically generated volumes.

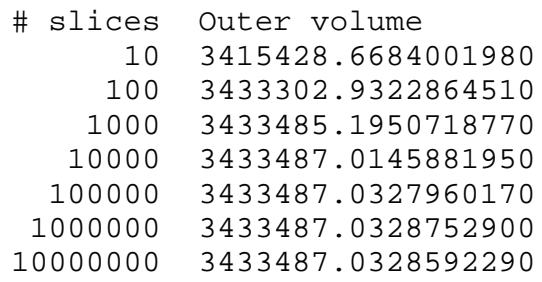

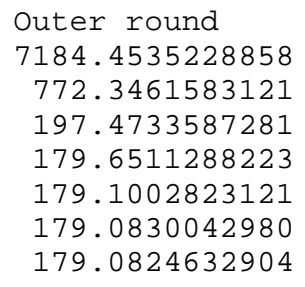

\author{
Inner round \\ 17.96674 \\ 9.55169 \\ 9.27450 \\ 9.26571 \\ 9.26544 \\ 9.26543 \\ 9.26543
}


A ProE model of this component was developed and is described in A ppendix B. Table 6-2 is a summary of the analytic, numerical, and ProE results. Generally, the results of all three methods agree with each other very well.

Table 6-2. Comparison of volumes by three different methods.

Volume element
Vtot (no step, no rnds)
Vtot (w/step, no rnds)
Step
Outer Round
Inner Round

ProE
553693.77
540499.08
-13194.69
-179.08
+9.26

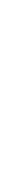

\begin{tabular}{cr} 
Numerical (1.0E7) & \multicolumn{1}{l}{ Analytic } \\
553693.76630 & 553693.76718 \\
540499.07716 & 540499.07803 \\
(N/A: exact) & -13194.68915 \\
-179.08246 & -179.08245 \\
+9.26543 & +9.26543
\end{tabular}


Analytic Solutions of an Unclassified Artifact 


\section{Chapter 7. Summary files and results}

This report has presented the details of the analytic definition of the unclassified test artifact. One of the main objectives of this work was to compare the results of this work to a highfidelity engineering model with the same definition. Such a model has been constructed and will be referred to as the "ProE model," after the name of the software package used to generate the model. Indeed, this model and the analytic definition provide exactly the same profile definitions to double-precision accuracy, with one notable exception.

It is extremely useful to utilize engineering models to define the shapes of complex objects. Unlike this problem, the shapes of general problems cannot be defined with analytic functions. Therefore it is extremely useful to be able to compare the engineering model with a problem where the exact definition is defined. While not conclusive, it would be a necessary prerequisite for such an engineering model to match exactly the analytic results, if it were to be used as a method for defining an experimental definition, for example.

\subsection{Analytic and ProE results}

Table 7-1 shows the results of the analytic model for four important points at an angle of theta $=60$ degrees. The four points correspond to the following points:

$c=$ the center of the probe sphere

$\mathrm{sp}=$ the spline point on the component surface

$\mathrm{ba}=$ the point on the probe sphere al ong the ray through the spline point

$\mathrm{cc}=$ the contact point where the probe sphere touches the profile

A t each of these points, the components may be defined for $x, y$, theta, and the radius. A Iso, for each 2-degree spline angle, the inner and outer profiles may be determined. Those 32 points for the 60-degree spline angle are shown in Table 7-1, for both the analytic method and the ProE model.

\subsection{Analysis of the results}

It is interesting to note that in Table 7-1, the results of the first three rows are nearly identical, i.e., the calculation of the probe sphere, the spline point, and the location on the probe sphere along the spline angle. Since the "cosine correction", as defined in Chapter 2, is simply the difference between the spline point radius and the projection of the contact radius al ong the spline angle $\left(R_{s p}-R_{b a}\right)$, those results are also very nearly identical, as shown in the last column of numbers. 

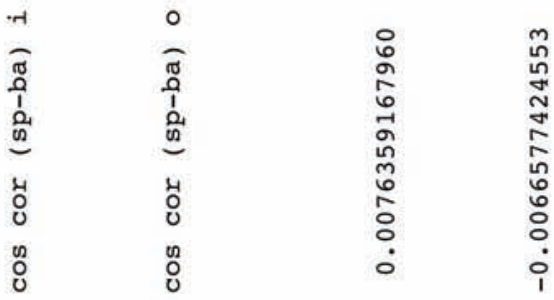

*

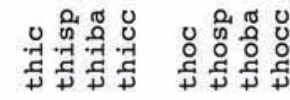

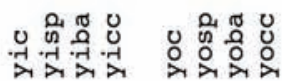

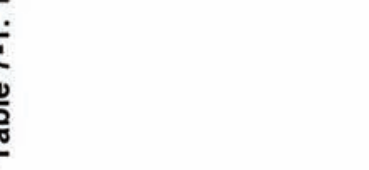

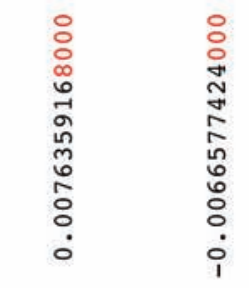
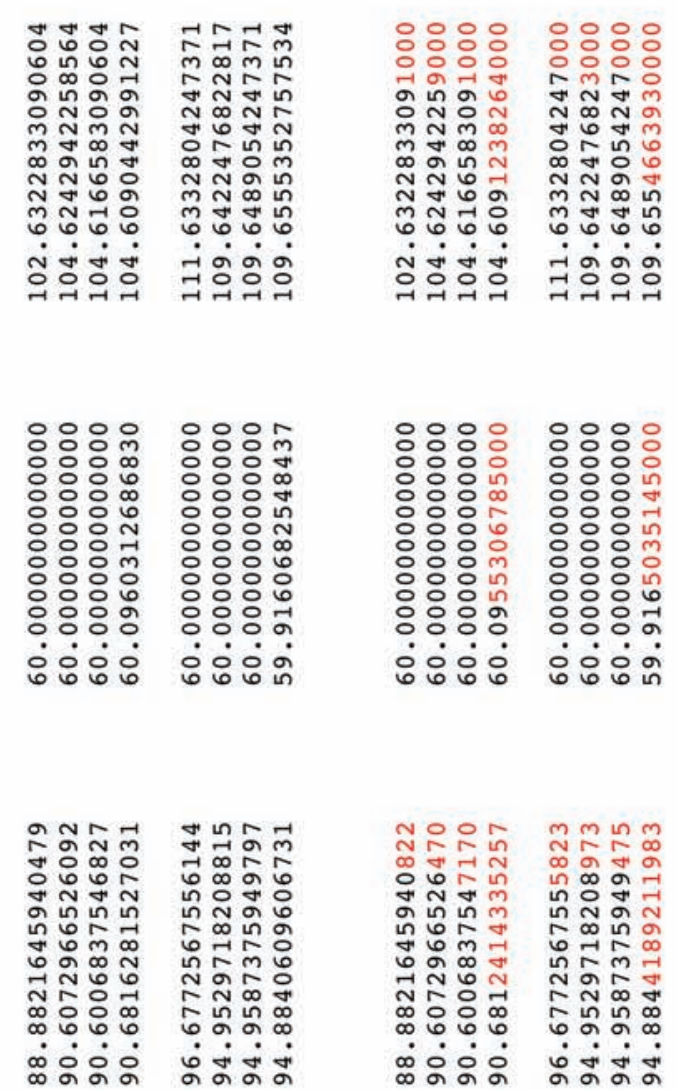

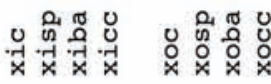

$\sqrt[3]{2}$

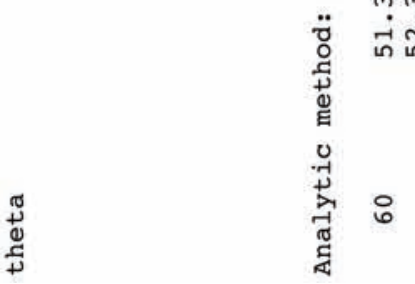

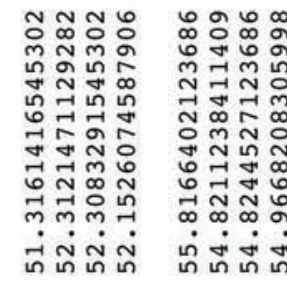

.

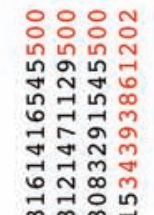

लेलेट्ले

ที่
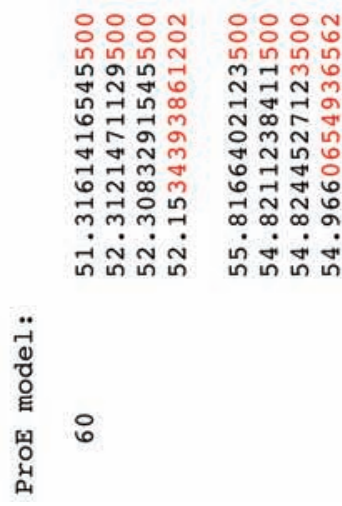

:

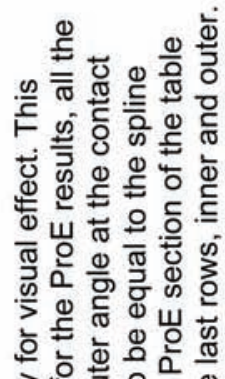

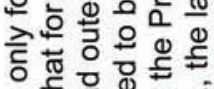

웡ㄷㅀ

응휴

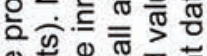

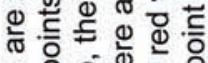

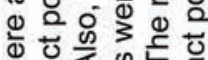

还

등웡웡등 엉 敋

结的贾.

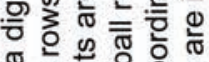

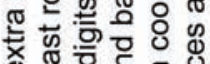

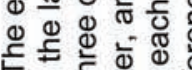

خ는

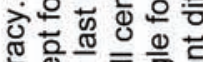

选

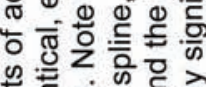

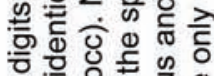

끈

诺

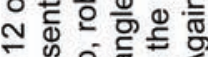

is क्ष

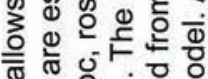

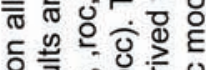

등

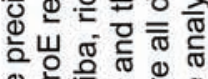

응흔은 월 응 둥은 올

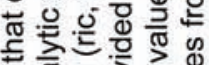

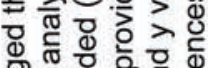

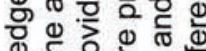

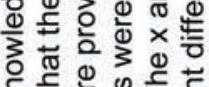
돈

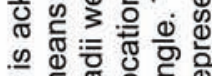

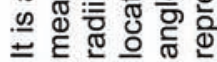


In the fourth row, however, the numbers begin to diverge from each other in the third or fourth decimal place, implying an error in the ProE model in the calculation of the location of the contact point. This apparent error is confirmed by a second analytic technique presented in $\mathrm{A}$ ppendix $\mathrm{C}$, which agrees identically with the analytic method presented in Chapters 1 to 6. M oreover, the error in the ProE model seems to increase from theta of zero to the maximum value of theta, approximately 96 degrees, as illustrated in Figure 7-1.

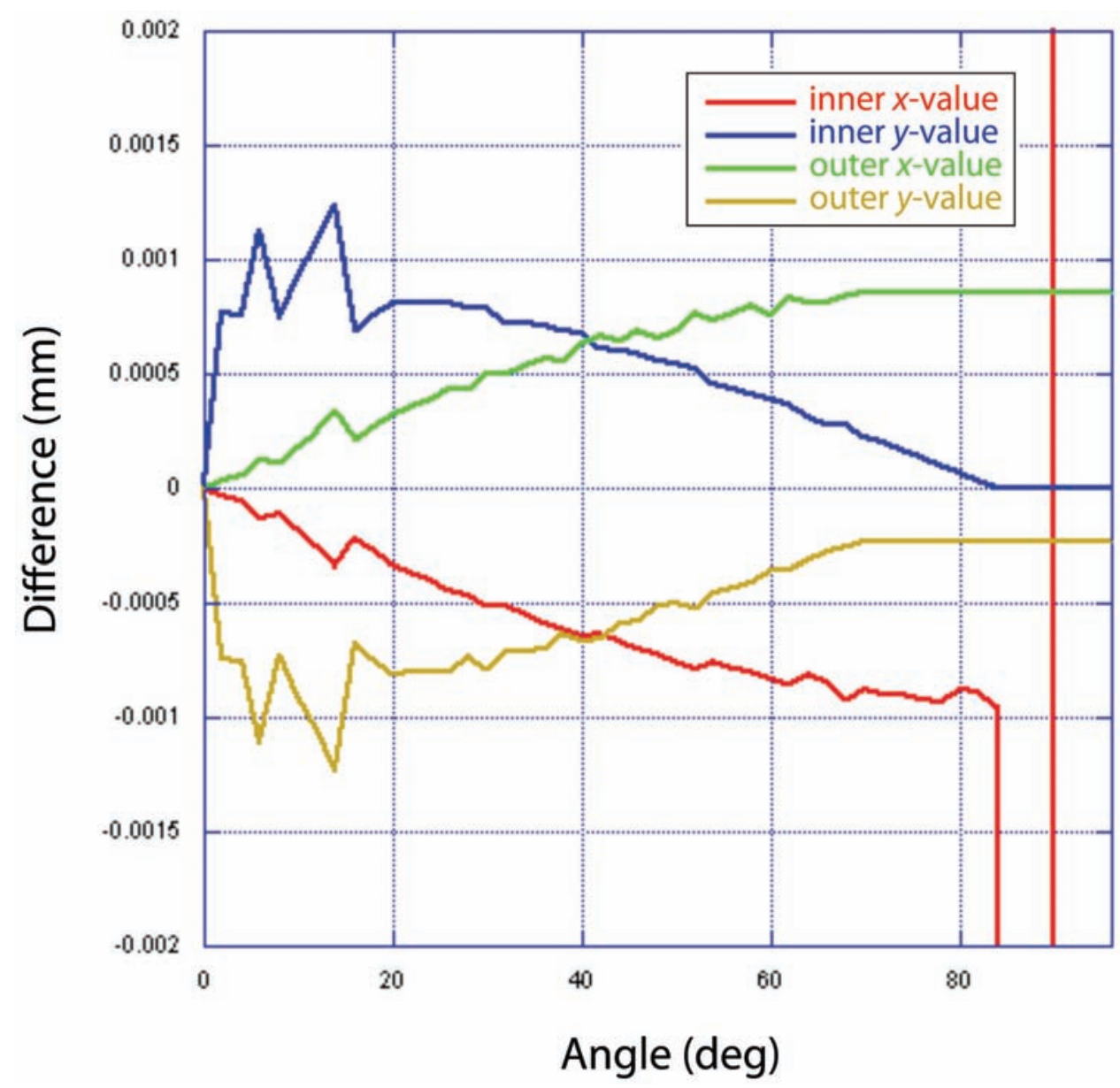

Figure 7-1. Differences between analytic and ProE values for $x$ and $y$ at the contact point.

Generally, the discrepancy between the two methods increases from zero as theta increases. There is a large jump in the inner $x$-value at 86 degrees that is unexplained at this time, but appears to be related to numbers that are wrong in the ProE model. For example, Table 7-2 shows that the angle and radius at theta equal to 90 degrees are wrong for the ProE model. The angle must be 90 degrees and the radius must be $100 \mathrm{~mm}$, by inspection. The analytic results are shown in Table 7-2 for comparison. 
Table 7-2. Angles and radii (with $x$ - and $y$-values below) for the inner and outer profiles at theta $=90$ degrees.
ind
thicca
xicca
ricca
thicca
rocca
xicca
yicca
xocca
yocca
Analytic Method:
$90 \quad 90.00000000000000$ 0.00000000000001
100.00000000000000
100.00000000000000
90.26386541096987
$-0.51359404262532 \quad 111.52110797632880$
ProE Model:
$90 \quad 90.00051507910000 \quad 100.00000000400000$
$-0.00089898262034 \quad 99.99999999995916$
$\begin{array}{rr}90.26430356250000 & 111.52252334510000 \\ -0.51444793894906 & 111.52133677721270\end{array}$

N ote, the third row in Table 7-2 shows that the ProE model is absolutely, positively wrong, as illustrated by the red digits. The inner radius and angle are $100 \mathrm{~mm}$ and 90 degrees, respectively, by inspection of Figure 1-1.

\subsection{Conclusion}

A nalytic solutions have been presented for the complete description of an unclassified test artifact. Comparisons to an engineering model have been made and some discrepancies observed. The differences apply to this analytic problem and do not necessarily imply errors in other models. It seems likely that the error in the software package is related to the lack of a "double-precision" implementation of one or more functions. It remains unresolved.

This document represents a small fraction of work that has been completed in support qualification processes necessary for a new inspection capability. That project is described in a document by J oshua M ontano, "CM M Shell Inspection (U)," LA-UR-11-02659. The following appendices provide additional detail for definition and support of the methods described in Chapters 1 to 7.

A ppendix A : Drawing of CM M test artifact

A ppendix B: ProE definition at 60 degrees (S. Schilling)

A ppendix C: A Iternate analytic solution (T. B eery)

A ppendix D: Cosine correction definition (L. Savage) 


\subsection{Acknowledgements}

$M$ any thanks are due to the reviewers of this work for their support and critical input. These individuals include Scott Schilling, J oshua M ontano, Tom B eery, Randy Flores, L owell Savage, M anikantan V elappan, M ario V aldez, L ucas V aldez, CJ Solomon, Nick Jenkins, and Rob Pelak. Their constant support is gratefully acknowledged.

Finally, thanks are due to $\mathrm{Craig}$ Carmer, without whose support this document would not have been possible. His contributions to the editing, composition, and style of the document, including his construction of all the figures (not to mention making all the equations look presentable), made the documentation of this work possible. 
Analytic Solutions of an Unclassified Artifact 


\section{A ppendix A. Drawing of C M M T est Artifact (157Y -700373)}

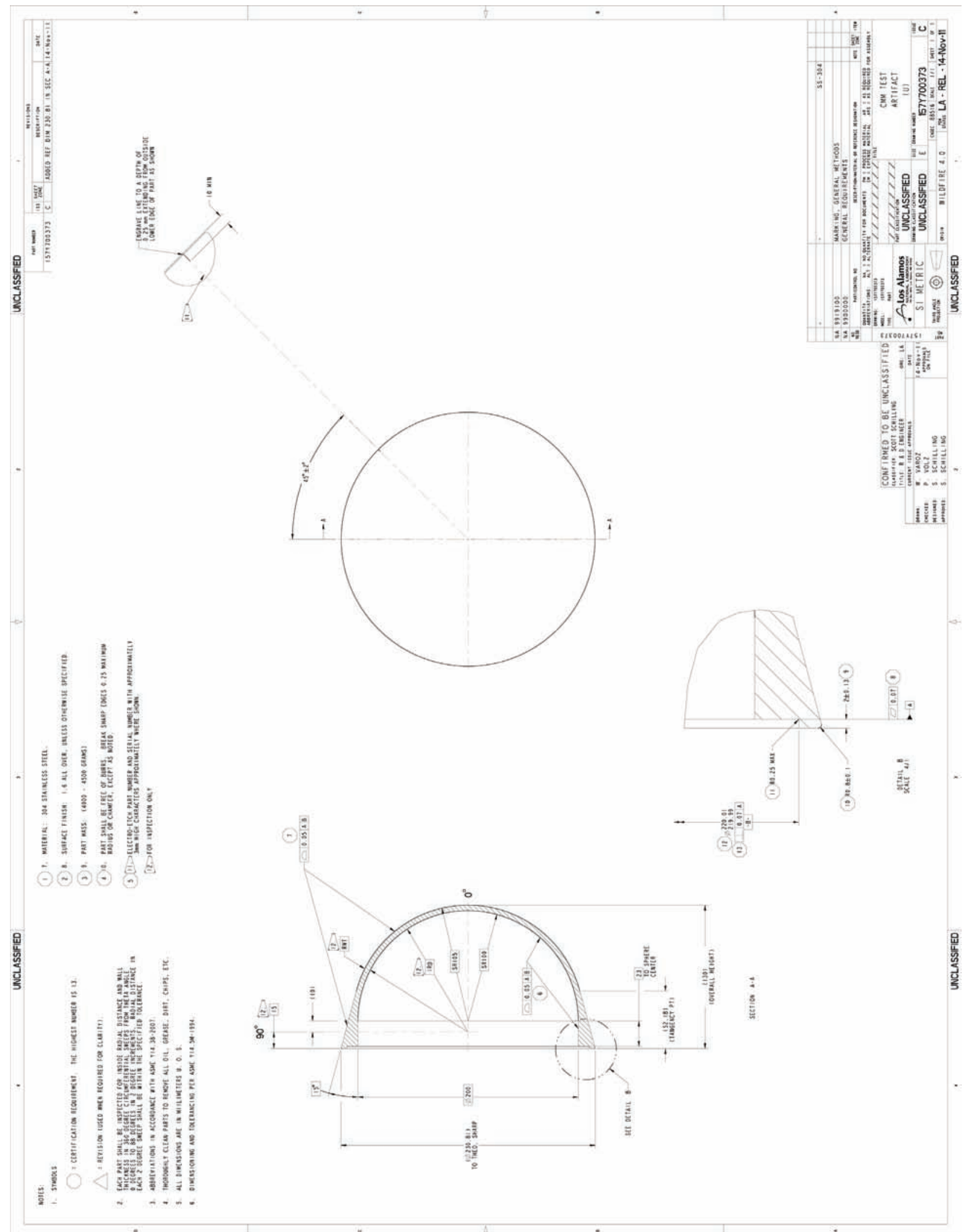


Analytic Solutions of an Unclassified Artifact 


\section{Appendix B. ProE definition at 60 degrees (Scott Schilling)}

Table B - 1 was provided as a guide to the analytic model. It was output from the early ProE model and it was expected to agree with the analytic results. The rows and columns agree with Table 7-1.

Table B-1. ProE definition at 60 degrees.

Inner profile at 60 degrees

$\mathrm{X}$

$\begin{array}{lll}51.31614165 & 88.88216459 & 60 \\ 52.31214711 & 90.60729665 & 60 \\ 52.30832915 & 90.60068375 & 60 \\ 52.15261513 & 90.68124459 & 60.09602665\end{array}$

Outer Profile at 60 degrees

Ball Center ID

Curve Ray ID

Ball Ray ID

Ball Curve ID

$\begin{array}{lll}55.81664021 & 96.67725676 & 60 \\ 54.82112384 & 94.95297182 & 60 \\ 54.82445271 & 94.95873759 & 60 \\ 54.96681360 & 94.88406439 & 59.91607242\end{array}$

111.6332804

109.6422477

109.6489054

109.6555346

Note that the number of significant figures in this table is fewer than in Table 7-1. The digits in red represent differences from the later ProE model. These differences are not completely understood at this time. Figures B-1 and B-2 are from the model.

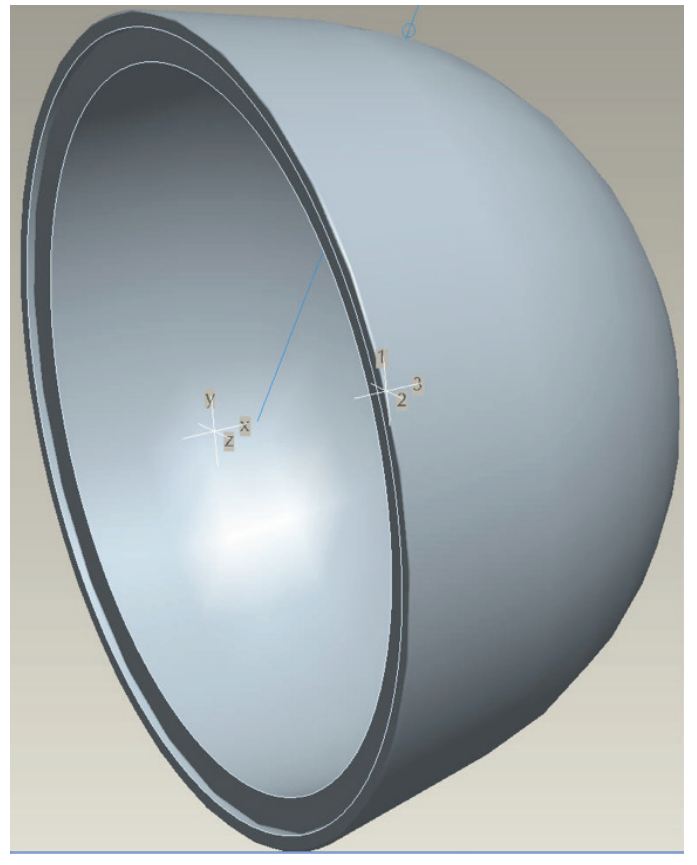

Figure B-1. ProE model image.

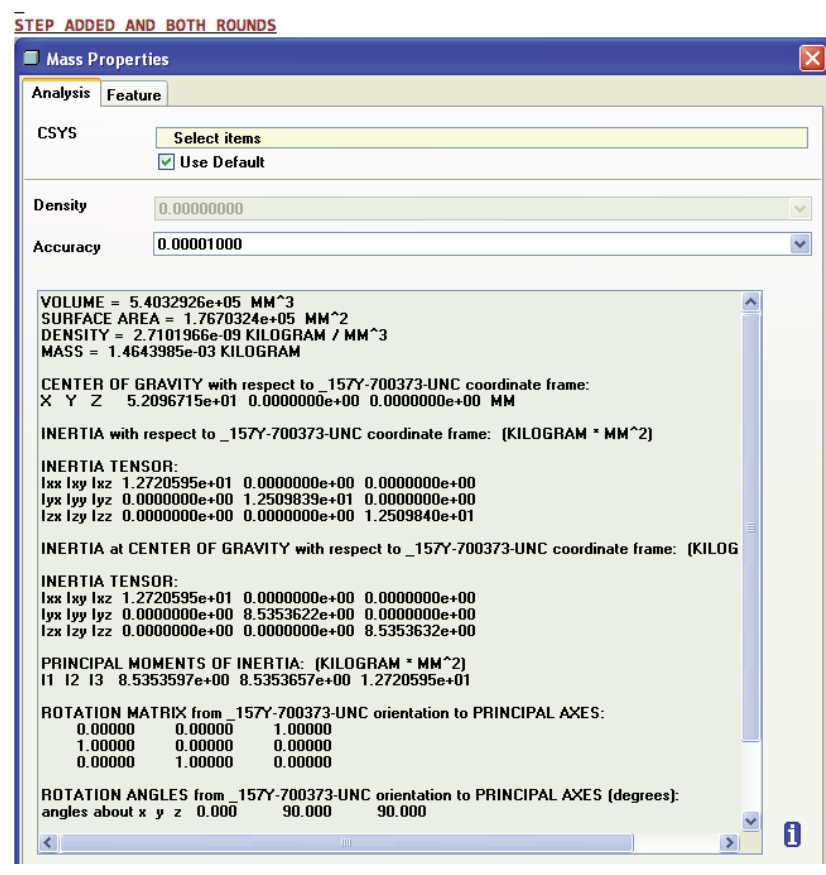

Figure B-2. Sample ProE output. 


\section{Appendix C . A Iternate analytic solution (Tom Beery)}

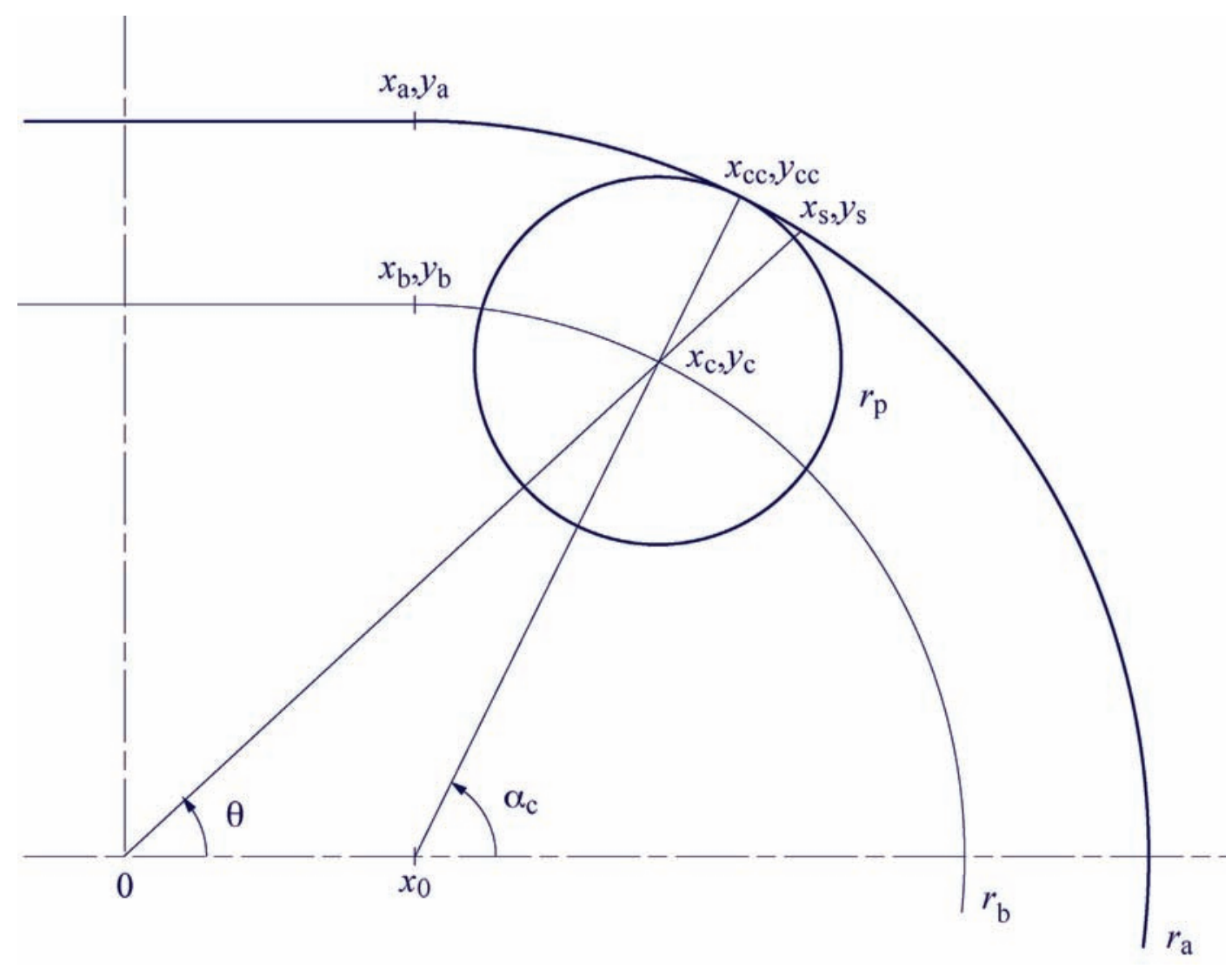

Figure C-1. Drawing for alternate solution of inner part contour.

\section{C-1 Graphical description}

The arc with a radius of $r_{b}$ is drawn. This represents all possible probe center locations while touching the outer arc.

A line is drawn through the measurement center using the measurement angle $\theta$. Where this line crosses the $r_{b}$ arc is the probe center $\left(x_{c}, y_{c}\right)$. The outer arc intersection is the spline point $\left(x_{5}, y_{s}\right)$. D rawing the probe circle is optional.

A line is drawn through the outer arc center $\left(x_{0}, 0\right)$, and the probe center. Where this line crosses the outer arc is the contact point $\left(\mathrm{x}_{\mathrm{cc}}, \mathrm{y}_{\mathrm{cc}}\right)$.

Solution of the straight line section proceeds in a similar manner. The contact point is found by drawing a line perpendicular to the section and passing though the probe center. 


\section{C-2 Summary of probe contact equations}

K nown variables:

$\theta$ : measurement angle

$r_{a}$ : surface radius

$r_{p}$ : probe tip radius

$\mathrm{x}_{0}$ : circle center

$\phi$ : angle of straight section

Solve for the probe contact center and point of contact.

$$
\begin{array}{ll}
r_{\mathrm{b}}=r_{\mathrm{a}}-r_{\mathrm{p}} & \text { radius of probe center } \\
\alpha_{\mathrm{c}}=\theta+\sin ^{-1}\left(\frac{x_{0} \sin \theta}{r_{\mathrm{b}}}\right) & \\
x_{\mathrm{c}}=x_{0}+r_{\mathrm{b}} \cos \alpha_{\mathrm{c}} & \text { probe center } \\
y_{\mathrm{c}}=r_{\mathrm{b}} \sin \alpha_{\mathrm{c}} & \\
x_{\mathrm{cc}}=x_{0}+\frac{r_{\mathrm{a}}\left(x_{\mathrm{c}}-x_{0}\right)}{r_{\mathrm{b}}} & \text { contact point } \\
y_{\mathrm{cc}}=\frac{r_{\mathrm{a}} y_{\mathrm{c}}}{r_{\mathrm{b}}} & \text { circle-line transition } \\
x_{\mathrm{b}}=x_{0}+r_{\mathrm{b}} \sin \phi & \\
y_{\mathrm{b}}=r_{\mathrm{b}} \cos \phi & \\
\theta_{\mathrm{b}}=\tan ^{-1}\left(\frac{y_{\mathrm{b}}}{x_{\mathrm{b}}}\right) & \text { line, point of contact } \\
x_{\mathrm{c}}=\frac{\left(y_{\mathrm{b}}+x_{\mathrm{b}} \tan \phi\right) \cos \theta}{\sin \theta+\tan \phi \cos \theta} & \text { line, probe center } \\
y_{\mathrm{c}}=y_{\mathrm{b}}+\left(x_{\mathrm{b}}-x_{\mathrm{c}}\right) \tan \phi & \\
x_{\mathrm{cc}}=x_{\mathrm{c}}+r_{\mathrm{p}} \sin \phi & \\
y_{\mathrm{cc}}=y_{\mathrm{c}}+r_{\mathrm{p}} \cos \phi &
\end{array}
$$

The spline point $\left(x_{s}, y_{s}\right)$ is a subset using $r_{a}$ in place of $r_{b}$. 
Table C-1 shows how this method compares to the analytic method presented in Chapters 1 through 7 and the ProE model results (from Table 7-1). The results are typical and are given only for an angle of 60 degrees.

Table C-1. Analytic results compared with ProE model at 60 degrees. Rc is the center of the probe sphere, Rs is the spline point on the component surface, and Rcc and $\theta \mathrm{cc}$ are the components of the contact points.

Inner profile at 60 degrees

\begin{tabular}{|c|c|c|c|c|}
\hline & $\mathrm{RC}$ & RS & $\mathrm{RCC}$ & $\theta c c$ \\
\hline Beery & 102.63228333897970 & 104.62429422585637 & 104.60904429935648 & 60.09603126721138 \\
\hline Trent & 102.6322833090604 & 104.6242942258564 & 104.6090442991227 & 60.0960312686830 \\
\hline Proe & 102.6322833091000 & 104.6242942259000 & 104.6091238264000 & 60.0955306785000 \\
\hline Oute. & profile at 60 & S & & \\
\hline Beery & 111.63328039483667 & 109.64224768228175 & 109.65553527555763 & 59.91606825608071 \\
\hline Trent & 111.6332804247371 & 109.6422476822817 & 109.6555352757534 & 59.9160682548437 \\
\hline ProE & 111.6332804247000 & 109.6422476823000 & 109.6554663930000 & 59.9165035145000 \\
\hline
\end{tabular}

The numbers in blue indicate a difference in the two analytic methods (B eery and Trent). Generally, all three methods provide identical results, to double precision accuracy (12 or 13 significant digits), with one exception. A s illustrated in Table 7-1, the agreement of the ProE model with both analytic methods is limited to 5 or 6 significant digits for the radius and the angle, $R_{c c}$ and $\theta_{c c}$, respectively, of the contact points (the last two columns). This indicates an accuracy problem in the ProE solution of the contact point location. In fact, if the center of the probe sphere and the spline point are known, it is straight-forward to calculate the contact point, given the simple geometric shapes that define the part.

It is a curiosity that the two analytic approaches summarized in Table C-1 show agreement between 15 and 10 significant places, representing accuracy beyond and below double precision. This discrepancy remains unresolved at this time. In all cases it is at least five orders of magnitude better agreement than the ProE model for the contact point location. 


\section{A ppendix D. Cosine correction definition (L owell Savage)}

Figure D-1 was provided to identify the fundamental characteristics of the mastering and measuring configurations. It provides the basis for the cosine correction used throughout this document.

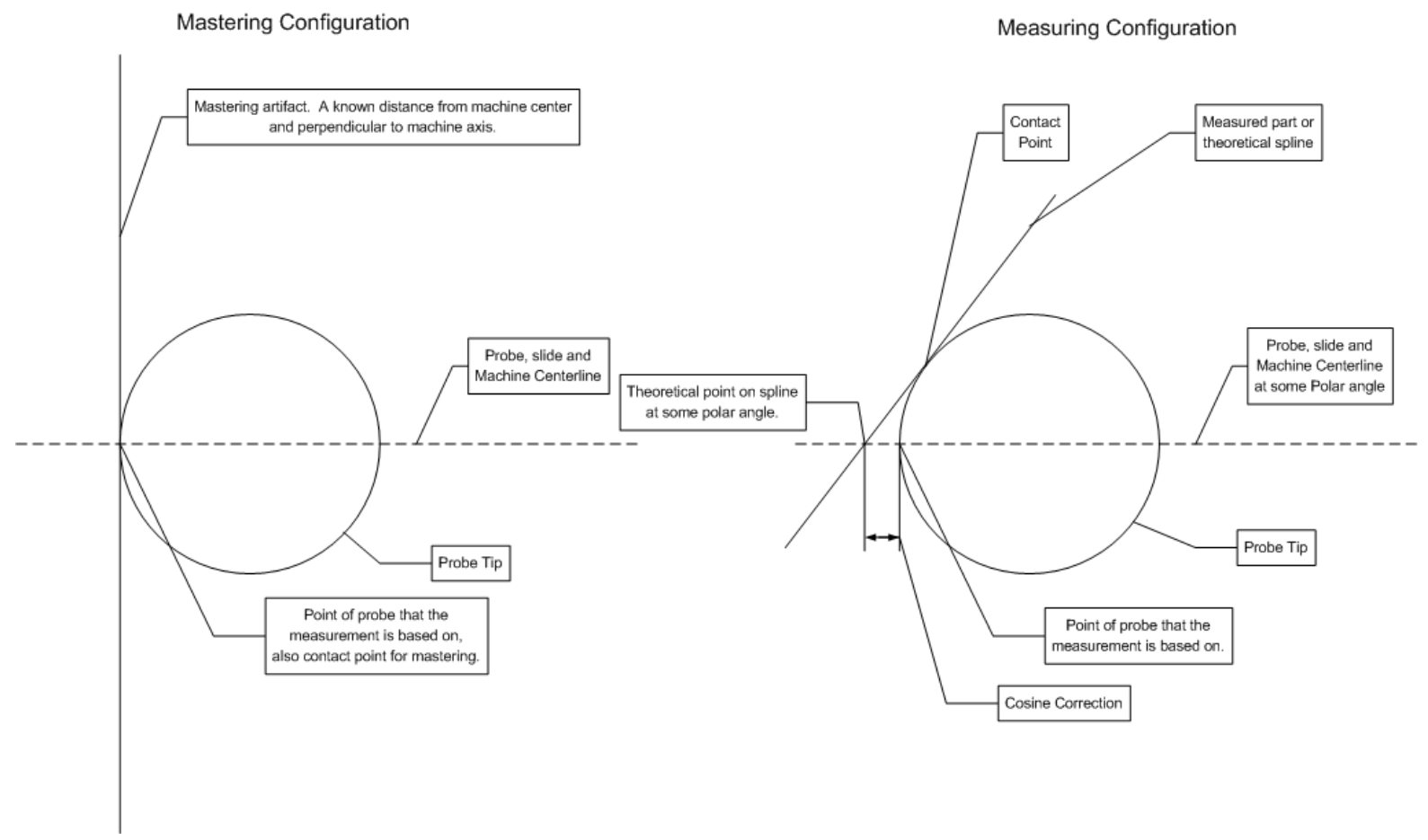

Figure D-1. Fundamental characteristics of the mastering and measuring configurations. 
Funding for this report was provided by the U.S. Department of Energy.

Edited by Craig Carmer of Group IRM-CAS.

Los Alamos National Laboratory, an Affirmative Action/ Equal Opportunity Employer, is operated by Los Alamos National Security, LLC, for the National Nuclear Security Administration of the U.S. Department of Energy under contract DE-AC52-06NA25396.

\section{INAS}

This report was prepared as an account of work sponsored by an agency of the U.S. Government. Neither Los Alamos National Security, LLC, the U.S. Government nor any agency thereof, nor any of their employees make any warranty, express or implied, or assume any legal liability or responsibility for the accuracy, completeness, or usefulness of any information, apparatus, product, or process disclosed, or represent that its use would not infringe privately owned rights. Reference herein to any specific commercial product, process, or service by trade name, trademark, manufacturer, or otherwise does not necessarily constitute or imply its endorsement, recommendation, or favoring by Los Alamos National Security, LLC, the U.S. Government, or any agency thereof. The views and opinions of authors expressed herein do not necessarily state or reflect those of Los Alamos National Security, LLC, the U.S. Government, or any agency thereof. Los Alamos National Laboratory strongly supports academic freedom and a researcher's right to publish; as an institution, however, the Laboratory does not endorse the viewpoint of a publication or guarantee its technical correctness. 
This report has been reproduced directly from the best available copy. It is available electronically on the Web (http://www.doe.gov/bridge).

Copies are available for sale to U.S. Department of Energy employees and contractors from:

Office of Scientific and Technical Information

P.O. Box 62

Oak Ridge, TN 37831

(865) 576-8401

Copies are available for sale to the public from: National Technical Information Service

U.S. Department of Commerce

5285 P ort Royal Road

Springfield, VA 22161

(800) 553-6847 


\section{Los Alamos NATIONAL LABORATORY}

